NBER WORKING PAPER SERIES

\title{
OPTIMAL MONETARY AND FISCAL POLICY: \\ A LINEAR-QUADRATIC APPROACH
}

\author{
Pierpaolo Benigno \\ Michael Woodford \\ Working Paper 9905 \\ http://www.nber.org/papers/w9905

\section{NATIONAL BUREAU OF ECONOMIC RESEARCH 1050 Massachusetts Avenue Cambridge, MA 02138}

August 2003

\begin{abstract}
We would like to thank Stefania Albanesi, Marios Angeletos, Albert Marcet, Ramon Marimon, seminar participants at New York University, Rutgers University, Universitat Pompeu Fabra, the Macro Annual conference, and the editors for helpful comments, Brad Strum and Vasco Curdia for research assistance, and the National Science Foundation for research support through a grant to the NBER. The views expressed herein are those of the authors and not necessarily those of the National Bureau of Economic Research.
\end{abstract}

C2003 by Pierpaolo Benigno and Michael Woodford. All rights reserved. Short sections of text, not to exceed two paragraphs, may be quoted without explicit permission provided that full credit, including (C) notice, is given to the source. 
Optimal Monetary and Fiscal Policy: A Linear-Quadratic Approach

Pierpaolo Benigno and Michael Woodford

NBER Working Paper No. 9905

August 2003

JEL No. E52, E61, E63

\section{$\underline{\text { ABSTRACT }}$}

We propose an integrated treatment of the problems of optimal monetary and fiscal policy, for an economy in which prices are sticky and the only available sources of government revenue are distorting taxes. Our linear-quadratic approach allows us to nest both conventional analyses of optimal monetary stabilization policy and analyses of optimal tax-smoothing as special cases of our more general framework. We show how a linear-quadratic policy problem can be derived which yields a correct linear approximation to the optimal policy rules from the point of view of the maximization of expected discounted utility in a dynamic stochastic general-equilibrium model. Finally, we derive targeting rules through which the monetary and fiscal authorities may implement the optimal equilibrium.

Pierpaolo Benigno

Department of Economics

New York University

269 Mercer Street

New York, NY 10003

pierpaolo.benigno@nyu.edu
Michael Woodford

Department of Economics

Princeton University

Princeton, NJ 08544

and NBER

woodford@princeton.edu 
While there are by now substantial literatures seeking to characterize optimal monetary and fiscal policy respectively, the two literatures have largely developed in isolation, and upon apparently contradictory foundations. The modern literature on dynamically optimal fiscal policy often abstracts from monetary aspects of the economy altogether, and so implicitly allows for no useful role for monetary policy. When monetary policy is considered within the theory of optimal fiscal policy, it is most often in the context of models with flexible prices; in these models, monetary policy matters only (i) because the level of nominal interest rates (and hence the opportunity cost of holding money) determines the size of certain distortions that result from the attempt to economize on money balances, and (ii) because the way in the price level varies in response to real disturbances determines the state-contingent real payoffs on (riskless) nominally-denominated government debt, which may facilitate tax-smoothing in the case that explicitly state-contingent debt is not available. The literature on optimal monetary policy has instead been mainly concerned with quite distinct objectives for monetary stabilization policy, namely the minimization of the distortions that result from prices or wages that do not adjust quickly enough to clear markets. At the same time, this literature typically ignores the fiscal consequences of alternative monetary policies; the characterizations of optimal monetary policy that are obtained are thus strictly correct only for a world in which lump-sum taxes are available.

Here we wish to consider the way in which the conclusions reached in each of these two familiar literatures must be modified if one takes simultaneous account of the basic elements of the policy problems addressed in each literature. On the one hand, we wish to consider how conventional conclusions with regard to the nature of an optimal monetary policy rule must be modified if one recognizes that the government's only sources of revenue are distorting taxes, so that the fiscal consequences of monetary policy matter for welfare. And on the other hand, we wish to consider how conventional conclusions with regard to optimal tax policy must be modified if one recognizes that prices do not instantaneously clear markets, so that output determination depends on aggregate demand, in addition to the supply-side factors stressed in the conventional theory of optimal taxation.

A number of recent papers have also sought to jointly consider optimal monetary and fiscal policy, in the context of models with sticky prices; important examples include Correia et al., (2001), Schmitt-Grohé and Uribe (2001), and Siu (2001). Our approach differs from those taken in these papers, however, in several respects. First, 
we model price stickiness in a different way than in any of these papers, namely, by assuming staggered pricing of the kind introduced by Calvo (1983). This particular form of price stickiness has been widely used both in analyses of optimal monetary policy in models with explicit microfoundations (e.g., Goodfriend and King, 1997; Clarida et al., 1999; Woodford, 2003) and in the empirical literature on optimizing models of the monetary transmission mechanism (e.g., Rotemberg and Woodford, 1997; Gali and Gertler, 1999; Sbordone, 2002).

Perhaps more importantly, we obtain analytical results rather than purely numerical ones. To obtain these results, we propose a linear-quadratic approach to the characterization of optimal monetary and fiscal policy, that allows us to nest both conventional analyses of optimal monetary policy, such as that of Clarida et al. (1999), and analyses of optimal tax-smoothing in the spirit of Barro (1979), Lucas and Stokey (1983), and Aiyagari et al. (2002) as special cases of our more general framework. We show how a linear-quadratic policy problem can be derived which yields a correct linear approximation to the optimal policy rules from the point of view of the maximization of expected discounted utility in a dynamic stochastic general-equilibrium model, building on the work of Benigno and Woodford (2003) for the case of optimal monetary policy when lump-sum taxes are available.

Finally, we do not content ourselves with merely characterizing the optimal dynamic responses of our policy instruments (and other state variables) to shocks under an optimal policy, given one assumption or another about the nature and statistical properties of the exogenous disturbances to our model economy. Instead, we also wish to derive policy rules that the monetary and fiscal authorities may reasonably commit themselves to follow, as a way of implementing the optimal equilibrium. In particular, we seek to characterize optimal policy in terms of optimal targeting rules for monetary and fiscal policy, of the kind proposed in the case of monetary policy by Svensson (1999), Svensson and Woodford (2003), and Giannoni and Woodford (2002, 2003). The rules are specified in terms of a target criterion for each authority; each authority commits itself to use its policy instrument each period in whatever way is necessary in order to allow it to project an evolution of the economy consistent with its target criterion. As discussed in Giannoni and Woodford (2002), we can derive rules of this form that are not merely consistent with the desired equilibrium responses to disturbances, but that in addition (i) imply a determinate rational-expectations equilibrium, so that there are not other equally possible (but less desirable) equilibria 
consistent with the same policy; and (ii) bring about optimal responses to shocks regardless of the character of and statistical properties of the exogenous disturbances in the model.

\section{The Policy Problem}

Here we describe our assumptions about the economic environment and pose the optimization problem that jointly optimal monetary and fiscal policies are intended to solve. The approximation method that we use to characterize the solution to this problem is then presented in the following section. Further details of the derivation of the structural equations of our model of nominal price rigidity can be found in Woodford (2003, chapter 3).

The goal of policy is assumed to be the maximization of the level of expected utility of a representative household. In our model, each household seeks to maximize

$$
U_{t_{0}} \equiv E_{t_{0}} \sum_{t=t_{0}}^{\infty} \beta^{t-t_{0}}\left[\tilde{u}\left(C_{t} ; \xi_{t}\right)-\int_{0}^{1} \tilde{v}\left(H_{t}(j) ; \xi_{t}\right) d j\right]
$$

where $C_{t}$ is a Dixit-Stiglitz aggregate of consumption of each of a continuum of differentiated goods,

$$
C_{t} \equiv\left[\int_{0}^{1} c_{t}(i)^{\frac{\theta}{\theta-1}} d i\right]^{\frac{\theta-1}{\theta}}
$$

with an elasticity of substitution equal to $\theta>1$, and $H_{t}(j)$ is the quantity supplied of labor of type $j$. Each differentiated good is supplied by a single monopolistically competitive producer. There are assumed to be many goods in each of an infinite number of "industries"; the goods in each industry $j$ are produced using a type of labor that is specific to that industry, and also change their prices at the same time. The representative household supplies all types of labor as well as consuming all types of goods. ${ }^{1}$ To simplify the algebraic form of our results, we restrict attention in this paper to the case of isoelastic functional forms,

$$
\tilde{u}\left(C_{t} ; \xi_{t}\right) \equiv \frac{C_{t}^{1-\tilde{\sigma}^{-1}} \bar{C}_{t}^{\tilde{\sigma}^{-1}}}{1-\tilde{\sigma}^{-1}}
$$

\footnotetext{
${ }^{1}$ We might alternatively assume specialization across households in the type of labor supplied; in the presence of perfect sharing of labor income risk across households, household decisions regarding consumption and labor supply would all be as assumed here.
} 


$$
\tilde{v}\left(H_{t} ; \xi_{t}\right) \equiv \frac{\lambda}{1+\nu} H_{t}^{1+\nu} \bar{H}_{t}^{-\nu}
$$

where $\tilde{\sigma}, \nu>0$, and $\left\{\bar{C}_{t}, \bar{H}_{t}\right\}$ are bounded exogenous disturbance processes. (We use the notation $\xi_{t}$ to refer to the complete vector of exogenous disturbances, including $\bar{C}_{t}$ and $\bar{H}_{t}$.)

We assume a common technology for the production of all goods, in which (industryspecific) labor is the only variable input,

$$
y_{t}(i)=A_{t} f\left(h_{t}(i)\right)=A_{t} h_{t}(i)^{1 / \phi},
$$

where $A_{t}$ is an exogenously varying technology factor, and $\phi>1$. Inverting the production function to write the demand for each type of labor as a function of the quantities produced of the various differentiated goods, and using the identity

$$
Y_{t}=C_{t}+G_{t}
$$

to substitute for $C_{t}$, where $G_{t}$ is exogenous government demand for the composite good, we can write the utility of the representative household as a function of the expected production plan $\left\{y_{t}(i)\right\} .^{2}$

We can furthermore express the relative quantities demanded of the differentiated goods each period as a function of their relative prices. This allows us to write the utility flow to the representative household in the form $U\left(Y_{t}, \Delta_{t} ; \xi_{t}\right)$, where

$$
\Delta_{t} \equiv \int_{0}^{1}\left(\frac{p_{t}(i)}{P_{t}}\right)^{-\theta(1+\omega)} d i \geq 1
$$

is a measure of price dispersion at date $t$, in which $P_{t}$ is the Dixit-Stiglitz price index

$$
P_{t} \equiv\left[\int_{0}^{1} p_{t}(i)^{1-\theta} d i\right]^{\frac{1}{1-\theta}},
$$

and the vector $\xi_{t}$ now includes the exogenous disturbances $G_{t}$ and $A_{t}$ as well as the preference shocks. Hence we can write our objective (1.1) as

$$
U_{t_{0}}=E_{t_{0}} \sum_{t=t_{0}}^{\infty} \beta^{t-t_{0}} U\left(Y_{t}, \Delta_{t} ; \xi_{t}\right) \text {. }
$$

${ }^{2}$ The government is assumed to need to obtain an exogenously given quantity of the Dixit-Stiglitz aggregate each period, and to obtain this in a cost-minimizing fashion. Hence the government allocates its purchases across the suppliers of differentiated goods in the same proportion as do households, and the index of aggregate demand $Y_{t}$ is the same function of the individual quantities $\left\{y_{t}(i)\right\}$ as $C_{t}$ is of the individual quantities consumed $\left\{c_{t}(i)\right\}$, defined in (1.2). 
The producers in each industry fix the prices of their goods in monetary units for a random interval of time, as in the model of staggered pricing introduced by Calvo (1983). We let $0 \leq \alpha<1$ be the fraction of prices that remain unchanged in any period. A supplier that changes its price in period $t$ chooses its new price $p_{t}(i)$ to maximize

$$
E_{t}\left\{\sum_{T=t}^{\infty} \alpha^{T-t} Q_{t, T} \Pi\left(p_{t}(i), p_{T}^{j}, P_{T} ; Y_{T}, \tau_{T}, \xi_{T}\right)\right\},
$$

where $Q_{t, T}$ is the stochastic discount factor by which financial markets discount random nominal income in period $T$ to determine the nominal value of a claim to such income in period $t$, and $\alpha^{T-t}$ is the probability that a price chosen in period $t$ will not have been revised by period $T$. In equilibrium, this discount factor is given by

$$
Q_{t, T}=\beta^{T-t} \frac{\tilde{u}_{c}\left(C_{T} ; \xi_{T}\right)}{\tilde{u}_{c}\left(C_{t} ; \xi_{t}\right)} \frac{P_{t}}{P_{T}} .
$$

The function $\Pi\left(p, p^{j}, P ; Y, \tau, \xi\right)$, defined in the appendix, indicates the after-tax nominal profits of a supplier with price $p$, in an industry with common price $p^{j}$, when the aggregate price index is equal to $P$, aggregate demand is equal to $Y$, and sales revenues are taxed at rate $\tau$. Profits are equal to after-tax sales revenues net of the wage bill, and the real wage demanded for labor of type $j$ is assumed to be given by

$$
w_{t}(j)=\mu_{t}^{w} \frac{\tilde{v}_{h}\left(H_{t}(j) ; \xi\right)}{\tilde{u}_{c}\left(C_{t} ; \xi_{t}\right)},
$$

where $\mu_{t}^{w} \geq 1$ is an exogenous markup factor in the labor market (allowed to vary over time, but assumed common to all labor markets), ${ }^{3}$ and firms are assumed to be wage-takers. We allow for wage markup variations in order to include the possibility of a "pure cost-push shock" that affects equilibrium pricing behavior while implying no change in the efficient allocation of resources. Note that variation in the tax rate $\tau_{t}$ has a similar effect on this pricing problem (and hence on supply behavior); this is the sole distortion associated with tax policy in the present model.

Each of the suppliers that revise their prices in period $t$ choose the same new price $p_{t}^{*}$. Under our assumed functional forms, the optimal choice has a closed-form solution

$$
\frac{p_{t}^{*}}{P_{t}}=\left(\frac{K_{t}}{F_{t}}\right)^{\frac{1}{1+\omega \theta}}
$$

\footnotetext{
${ }^{3}$ In the case that we assume that $\mu_{t}^{w}=1$ at all times, our model is one in which both households and firms are wage-takers, or there is efficient contracting between them.
} 
where $\omega \equiv \phi(1+\nu)-1>0$ is the elasticity of real marginal cost in an industry with respect to industry output, and $F_{t}$ and $K_{t}$ are functions of current aggregate output $Y_{t}$, the current tax rate $\tau_{t}$, the current exogenous state $\xi_{t}{ }^{4}$ and the expected future evolution of inflation, output, taxes and disturbances, defined in the appendix.

The price index then evolves according to a law of motion

$$
P_{t}=\left[(1-\alpha) p_{t}^{* 1-\theta}+\alpha P_{t-1}^{1-\theta}\right]^{\frac{1}{1-\theta}},
$$

as a consequence of (1.4). Substitution of (1.9) into (1.10) implies that equilibrium inflation in any period is given by

$$
\frac{1-\alpha \Pi_{t}^{\theta-1}}{1-\alpha}=\left(\frac{F_{t}}{K_{t}}\right)^{\frac{\theta-1}{1+\omega \theta}}
$$

where $\Pi_{t} \equiv P_{t} / P_{t-1}$. This defines a short-run aggregate supply relation between inflation and output, given the current tax rate $\tau_{t}$, current disturbances $\xi_{t}$, and expectations regarding future inflation, output, taxes and disturbances. Because the relative prices of the industries that do not change their prices in period $t$ remain the same, we can also use (1.10) to derive a law of motion of the form

$$
\Delta_{t}=h\left(\Delta_{t-1}, \Pi_{t}\right)
$$

for the dispersion measure defined in (1.3). This is the source in our model of welfare losses from inflation or deflation.

We abstract here from any monetary frictions that would account for a demand for central-bank liabilities that earn a substandard rate of return; we nonetheless assume that the central bank can control the riskless short-term nominal interest rate $i_{t}{ }^{5}$ which is in turn related to other financial asset prices through the arbitrage relation

$$
1+i_{t}=\left[E_{t} Q_{t, t+1}\right]^{-1}
$$

We shall assume that the zero lower bound on nominal interest rates never binds under the optimal policies considered below, ${ }^{6}$ so that we need not introduce any

\footnotetext{
${ }^{4}$ The disturbance vector $\xi_{t}$ is now understood to include the current value of the wage markup $\mu_{t}^{w}$.

${ }^{5}$ For discussion of how this is possible even in a "cashless" economy of the kind assumed here, see Woodford (2003, chapter 2).

${ }^{6}$ This can be shown to be true in the case of small enough disturbances, given that the nominal interest rate is equal to $\bar{r}=\beta^{-1}-1>0$ under the optimal policy in the absence of disturbances.
} 
additional constraint on the possible paths of output and prices associated with a need for the chosen evolution of prices to be consistent with a non-negative nominal interest rate.

Our abstraction from monetary frictions, and hence from the existence of seignorage revenues, does not mean that monetary policy has no fiscal consequences, for interest-rate policy and the equilibrium inflation that results from it have implications for the real burden of government debt. For simplicity, we shall assume that all public debt consists of riskless nominal one-period bonds. The nominal value $B_{t}$ of end-of-period public debt then evolves according to a law of motion

$$
B_{t}=\left(1+i_{t-1}\right) B_{t-1}+P_{t} s_{t}
$$

where the real primary budget surplus is given by

$$
s_{t} \equiv \tau_{t} Y_{t}-G_{t}-\zeta_{t}
$$

Here $\tau_{t}$, the share of the national product that is collected by the government as tax revenues in period $t$, is the key fiscal policy decision each period; the real value of (lump-sum) government transfers $\zeta_{t}$ is treated as exogenously given, as are government purchases $G_{t}$. (We introduce the additional type of exogenously given fiscal needs so as to be able to analyze the consequences of a "purely fiscal" disturbance, with no implications for the real allocation of resources beyond those that follow from its effect on the government budget.)

Rational-expectations equilibrium requires that the expected path of government surpluses must satisfy an intertemporal solvency condition

$$
b_{t-1} \frac{P_{t-1}}{P_{t}}=E_{t} \sum_{T=t}^{\infty} R_{t, T} s_{T}
$$

in each state of the world that may be realized at date $t,{ }^{7}$ where $R_{t, T} \equiv Q_{t, T} P_{T} / P_{t}$ is the stochastic discount factor for a real income stream, and This condition restricts

\footnotetext{
${ }^{7}$ See Woodford (2003, chapter 2) for derivation of this condition from household optimization together with market clearing. The condition should not be interpreted as an a priori constraint on possible government policy rules, as discussed in Woodford (2001). However, when we consider the problem of choosing an optimal plan from the among the possible rational-expectations equilibria, this condition must be imposed among the constraints on the set of equilibria that one may hope to bring about.
} 
the possible paths that may be chosen for the tax rate $\left\{\tau_{t}\right\}$. Monetary policy can affect this constraint, however, both by affecting the period $t$ inflation rate (which affects the left-hand side) and (in the case of sticky prices) by affecting the discount factors $\left\{R_{t, T}\right\}$.

Under the standard (Ramsey) approach to the characterization of an optimal policy commitment, one chooses among state-contingent paths $\left\{\Pi_{t}, Y_{t}, \tau_{t}, b_{t}, \Delta_{t}\right\}$ from some initial date $t_{0}$ onward that satisfy (1.11), (1.12), and (1.15) for each $t \geq t_{0}$, given initial government debt $b_{t_{0}-1}$ and price dispersion $\Delta_{t_{0}-1}$, so as to maximize (1.5). Such a $t_{0}$-optimal plan requires commitment, insofar as the corresponding $t$-optimal plan for some later date $t$, given the conditions $b_{t-1}, \Delta_{t-1}$ obtaining at that date, will not involve a continuation of the $t_{0}$-optimal plan. This failure of time consistency occurs because the constraints on what can be achieved at date $t_{0}$, consistent with the existence of a rational-expectations equilibrium, depend on the expected paths of inflation, output and taxes at later dates; but in the absence of a prior commitment, a planner would have no motive at those later dates to choose a policy consistent with the anticipations that it was desirable to create at date $t_{0}$.

However, the degree of advance commitment that is necessary to bring about an optimal equilibrium is of only a limited sort. Let

$$
W_{t} \equiv E_{t} \sum_{T=t}^{\infty} \beta^{T-t} \tilde{u}_{c}\left(Y_{T}-G_{T} ; \xi_{T}\right) s_{T},
$$

and let $\mathcal{F}$ be the set of values for $\left(b_{t-1}, \Delta_{t-1}, F_{t}, K_{t}, W_{t}\right)$ such that there exist paths $\left\{\Pi_{T}, Y_{T}, \tau_{T}, b_{T}, \Delta_{T}\right\}$ for dates $T \geq t$ that satisfy (1.11), (1.12), and (1.15) for each $T$, that are consistent with the specified values for $F_{t}, K_{t}$, and $W_{t}$, and that imply a well-defined value for the objective $U_{t}$ defined in (1.5). Furthermore, for any $\left(b_{t-1}, \Delta_{t-1}, F_{t}, K_{t}, W_{t}\right) \in \mathcal{F}$, let $V\left(b_{t-1}, \Delta_{t-1}, X_{t} ; \xi_{t}\right)$ denote the maximum attainable value of $U_{t}$ among the state-contingent paths that satisfy the constraints just mentioned, where $X_{t} \equiv\left(F_{t}, K_{t}, W_{t}\right){ }^{8}$ Then the $t_{0}$-optimal plan can be obtained as the solution to a two-stage optimization problem, as shown in the appendix.

In the first stage, values of the endogenous variables $x_{t_{0}}$, where $x_{t} \equiv\left(\Pi_{t}, Y_{t}, \tau_{t}, b_{t}, \Delta_{t}\right)$, and state-contingent commitments $X_{t_{0}+1}\left(\xi_{t_{0}+1}\right)$ for the following period, are chosen,

\footnotetext{
${ }^{8}$ In our notation for the value function $V, \xi_{t}$ denotes not simply the vector of disturbances in period $t$, but all information in period $t$ about current and future disturbances. This corresponds to the disturbance vector $\xi_{t}$ referred to earlier in the case that the disturbance vector follows a Markov process.
} 
subject to a set of constraints stated in the appendix, including the requirement that the choices $\left(b_{t_{0}}, \Delta_{t_{0}}, X_{t_{0}+1}\right) \in \mathcal{F}$ for each possible state of the world $\xi_{t_{0}+1}$. These variables are chosen so as to maximize the objective $\hat{J}\left[x_{t_{0}}, X_{t_{0}+1}(\cdot)\right]\left(\xi_{t_{0}}\right)$, where we define the functional

$$
\hat{J}\left[x_{t}, X_{t+1}(\cdot)\right]\left(\xi_{t}\right) \equiv U\left(Y_{t}, \Delta_{t} ; \xi_{t}\right)+\beta E_{t} V\left(b_{t}, \Delta_{t}, X_{t+1} ; \xi_{t+1}\right)
$$

In the second stage, the equilibrium evolution from period $t_{0}+1$ onward is chosen to solve the maximization problem that defines the value function $V\left(b_{t_{0}}, \Delta_{t_{0}}, X_{t_{0}+1} ; \xi_{t_{0}+1}\right)$, given the state of the world $\xi_{t_{0}+1}$ and the precommitted values for $X_{t_{0}+1}$ associated with that state. The key to this result is a demonstration that there are no restrictions on the evolution of the economy from period $t_{0}+1$ onward that are required in order for this expected evolution to be consistent with the values chosen for $x_{t_{0}}$, except consistency with the commitments $X_{t_{0}+1}\left(\xi_{t_{0}+1}\right)$ chosen in the first stage.

The optimization problem in stage two of this reformulation of the Ramsey problem is of the same form as the Ramsey problem itself, except that there are additional constraints associated with the precommitted values for the elements of $X_{t_{0}+1}\left(\xi_{t_{0}+1}\right)$. Let us consider a problem like the Ramsey problem just defined, looking forward from some period $t_{0}$, except under the constraints that the quantities $X_{t_{0}}$ must take certain given values, where $\left(b_{t_{0}-1}, \Delta_{t_{0}-1}, X_{t_{0}}\right) \in \mathcal{F}$. This constrained problem can similarly be expressed as a two-stage problem of the same form as above, with an identical stage two problem to the one described above. Stage two of this constrained problem is thus of exactly the same form as the problem itself. Hence the constrained problem has a recursive form. It can be decomposed into an infinite sequence of problems, in which in each period $t,\left(x_{t}, X_{t+1}(\cdot)\right)$ are chosen to maximize $\hat{J}\left[x_{t}, X_{t+1}(\cdot)\right]\left(\xi_{t}\right)$, subject to the constraints of the stage one problem, given the predetermined state variables $\left(b_{t-1}, \Delta_{t-1}\right)$ and the precommitted values $X_{t}$.

Our aim here is to characterize policy that solves this constrained optimization problem (stage two of the original Ramsey problem), i.e., policy that is optimal from some date $t$ onward given precommitted values for $X_{t}$. Because of the recursive form of this problem, it is possible for a commitment to a time-invariant policy rule from date $t$ onward to implement an equilibrium that solves the problem, for some specification of the initial commitments $X_{t}$. A time-invariant policy rule with this property is said by Woodford (2003, chapter 7 ) to be "optimal from a timeless perspective." 9 Such

\footnotetext{
${ }^{9}$ See also Woodford (1999) and Giannoni and Woodford (2002).
} 
a rule is one that a policymaker that solves a traditional Ramsey problem would be willing to commit to eventually follow, though the solution to the Ramsey problem involves different behavior initially, as there is no need to internalize the effects of prior anticipation of the policy adopted for period $t_{0} \cdot{ }^{10}$ One might also argue that it is desirable to commit to follow such a rule immediately, even though such a policy would not solve the (unconstrained) Ramsey problem, as a way of demonstrating one's willingness to accept constraints that one wishes the public to believe that one will accept in the future.

\section{A Linear-Quadratic Approximate Problem}

In fact, we shall here characterize the solution to this problem (and similarly, derive optimal time-invariant policy rules) only for initial conditions near certain steadystate values, allowing us to use local approximations in characterizing optimal policy. ${ }^{11}$ We establish that these steady-state values have the property that if one starts from initial conditions close enough to the steady state, and exogenous disturbances thereafter are small enough, the optimal policy subject to the initial commitments remains forever near the steady state. Hence our local characterization would describe the long run character of Ramsey policy, in the event that disturbances are small enough, and that deterministic Ramsey policy would converge to the steady state. $^{12}$ Of greater interest here, it describes policy that is optimal from a timeless perspective in the event of small disturbances.

We first must show the existence of a steady state, i.e., of an optimal policy (under

\footnotetext{
${ }^{10}$ For example, in the case of positive initial nominal government debt, the $t_{0}$-optimal policy would involve a large inflation in period $t_{0}$, in order to reduce the pre-existing debt burden, but a commitment not to respond similarly to the existence of nominal government debt in later periods.

${ }^{11}$ Local approximations of the same sort are often used in the literature in numerical characterizations of Ramsey policy. Strictly speaking, however, such approximations are valid only in the case of initial commitments $X_{t_{0}}$ near enough to the steady-state values of these variables, and the $t_{0}$-optimal (Ramsey) policy need not involve values of $X_{t_{0}}$ near the steady-state values, even in the absence of random disturbances.

${ }^{12}$ Benigno and Woodford (2003) gives an example of an application in which Ramsey policy does converge asymptotically to the steady state, so that the solution to the approximate problem approximates the response to small shocks under the Ramsey policy, at dates long enough after $t_{0}$. We cannot make a similar claim in the present application, however, because of the unit root in the dynamics associated with optimal policy.
} 
appropriate initial conditions) that involves constant values of all variables. To this end we consider the purely deterministic case, in which the exogenous disturbances $\bar{C}_{t}, G_{t}, \bar{H}_{t}, A_{t}, \mu_{t}^{w}, \zeta_{t}$ each take constant values $\bar{C}, \bar{G}, \bar{H}, \bar{A}, \bar{\mu}^{w}>0$ and $\bar{\zeta} \geq 0$ for all $t \geq t_{0}$, and assume an initial real public debt $b_{t_{0}-1}=\bar{b}>0$. We wish to find an initial degree of price dispersion $\Delta_{t_{0}-1}$ and initial commitments $X_{t_{0}}=\bar{X}$ such that the solution to the "stage two" problem defined above involves a constant policy $x_{t}=\bar{x}, X_{t+1}=\bar{X}$ each period, in which $\bar{b}$ is equal to the initial real debt and $\bar{\Delta}$ is equal to the initial price dispersion. We show in the appendix that the first-order conditions for this problem admit a steady-state solution of this form, and we verify below that the second-order conditions for a local optimum are also satisfied.

Regardless of the initial public debt $\bar{b}$, we show that $\bar{\Pi}=1$ (zero inflation), and correspondingly that $\bar{\Delta}=1$ (zero price dispersion). Note that our conclusion that the optimal steady-state inflation rate is zero generalizes the result of Benigno and Woodford (2003) for the case in which taxes are lump-sum at the margin. We may furthermore assume without loss of generality that the constant values of $\bar{C}$ and $\bar{H}$ are chosen (given the initial government debt $\bar{b}$ ) so that in the optimal steady state, $C_{t}=\bar{C}$ and $H_{t}=\bar{H}$ each period. ${ }^{13}$ The associated steady-state tax rate is given by

$$
\bar{\tau}=s_{G}+\frac{\bar{\zeta}+(1-\beta) \bar{b}}{\bar{Y}},
$$

where $\bar{Y}=\bar{C}+\bar{G}>0$ is the steady-state output level, and $s_{G} \equiv \bar{G} / \bar{Y}<1$ is the steady-state share of output purchased by the government. As shown in the appendix, this solution necessarily satisfies $0<\bar{\tau}<1$.

We next wish to characterize the optimal responses to small perturbations of the initial conditions and small fluctuations in the disturbance processes around the above values. To do this, we compute a linear-quadratic approximate problem, the solution to which represents a linear approximation to the solution to the "stage two" policy problem, using the method introduced in Benigno and Woodford (2003). An important advantage of this approach is that it allows direct comparison of our results with those obtained in other analyses of optimal monetary stabilization policy. Other advantages are that it makes it straightforward to verify whether the second-order conditions hold that are required in order for a solution to our first-order conditions to be at least a local optimum, ${ }^{14}$ and that it provides us with a welfare measure with

\footnotetext{
${ }^{13}$ Note that we may assign arbitrary positive values to $\bar{C}, \bar{H}$ without changing the nature of the implied preferences, as long as the value of $\lambda$ is appropriately adjusted.
} 
which to rank alternative sub-optimal policies, in addition to allowing computation of the optimal policy.

We begin by computing a Taylor-series approximation to our welfare measure (1.5), expanding around the steady-state allocation defined above, in which $y_{t}(i)=\bar{Y}$ for each good at all times and $\xi_{t}=0$ at all times. ${ }^{15}$ As a second-order (logarithmic) approximation to this measure, we obtain ${ }^{16}$

$$
\begin{aligned}
U_{t_{0}} & =\bar{Y} \bar{u}_{c} \cdot E_{t_{0}} \sum_{t=t_{0}}^{\infty} \beta^{t-t_{0}} \Phi \widehat{Y}_{t}-\frac{1}{2} u_{y y} \widehat{Y}_{t}^{2}+\widehat{Y}_{t} u_{\xi} \xi_{t}-u_{\Delta} \hat{\Delta}_{t} \\
& + \text { t.i.p. }+\mathcal{O}\left(\|\xi\|^{3}\right),
\end{aligned}
$$

where $\hat{Y}_{t} \equiv \log \left(Y_{t} / \bar{Y}\right)$ and $\hat{\Delta}_{t} \equiv \log \Delta_{t}$ measure deviations of aggregate output and the price dispersion measure from their steady-state levels, the term "t.i.p." collects terms that are independent of policy (constants and functions of exogenous disturbances) and hence irrelevant for ranking alternative policies, and $\|\xi\|$ is a bound on the amplitude of our perturbations of the steady state. ${ }^{17}$ Here the coefficient

$$
\Phi \equiv 1-\frac{\theta-1}{\theta} \frac{1-\bar{\tau}}{\bar{\mu}^{w}}<1
$$

measures the steady-state wedge between the marginal rate of substitution between consumption and leisure and the marginal product of labor, and hence the inefficiency of the steady-state output level $\bar{Y}$. Under the assumption that $\bar{b}>0$, we necessarily

\footnotetext{
${ }^{14}$ Benigno and Woodford (2003) show that these conditions can fail to hold, so that a small amount of arbitrary randomization of policy is welfare-improving, but argue that the conditions under which this occurs in their model are not empirically plausible.

${ }^{15}$ Here the elements of $\xi_{t}$ are assumed to be $\bar{c}_{t} \equiv \log \left(\bar{C}_{t} / \bar{C}\right), \bar{h}_{t} \equiv \log \left(\bar{H}_{t} / \bar{H}\right), a_{t} \equiv \log \left(A_{t} / \bar{A}\right), \hat{\mu}_{t}^{w} \equiv$ $\log \left(\mu_{t}^{w} / \bar{\mu}^{w}\right), \hat{G}_{t} \equiv\left(G_{t}-\bar{G}\right) / \bar{Y}$, and $\hat{\zeta}_{t} \equiv\left(\zeta_{t}-\bar{\zeta}\right) / \bar{Y}$, so that a value of zero for this vector corresponds to the steady-state values of all disturbances. The perturbations $\hat{G}_{t}$ and $\zeta_{t}$ are not defined to be logarithmic so that we do not have to assume positive steady-state values for these variables.

${ }^{16}$ See the appendix for details. Our calculations here follow closely those of Woodford (2003, chapter 6) and Benigno and Woodford (2003).

${ }^{17}$ Specifically, we use the notation $\mathcal{O}\left(\|\xi\|^{k}\right)$ as shorthand for $\mathcal{O}\left(\left\|\xi, \hat{b}_{t_{0}-1}, \hat{\Delta}_{t_{0}-1}^{1 / 2}, \hat{X}_{t_{0}}\right\|^{k}\right)$, where in each case hats refer to log deviations from the steady-state values of the various parameters of the policy problem. We treat $\hat{\Delta}_{t_{0}}^{1 / 2}$ as an expansion parameter, rather than $\hat{\Delta}_{t_{0}}$ because (1.12) implies that deviations of the inflation rate from zero of order $\epsilon$ only result in deviations in the dispersion measure $\Delta_{t}$ from one of order $\epsilon^{2}$. We are thus entitled to treat the fluctuations in $\Delta_{t}$ as being only of second order in our bound on the amplitude of disturbances, since if this is true at some initial date it will remain true thereafter.
} 
have $\Phi>0$, meaning that steady-state output is inefficiently low. The coefficients $u_{y y}, u_{\xi}$ and $u_{\Delta}$ are defined in the appendix.

Under the Calvo assumption about the distribution of intervals between price changes, we can relate the dispersion of prices to the overall rate of inflation, allowing us to rewrite (2.1) as

$$
\begin{aligned}
U_{t_{0}} & =\bar{Y} \bar{u}_{c} \cdot E_{t_{0}} \sum_{t=t_{0}}^{\infty} \beta^{t-t_{0}}\left[\Phi \widehat{Y}_{t}-\frac{1}{2} u_{y y} \widehat{Y}_{t}^{2}+\widehat{Y}_{t} u_{\xi} \xi_{t}-u_{\pi} \pi_{t}^{2}\right] \\
& + \text { t.i.p. }+\mathcal{O}\left(\|\xi\|^{3}\right),
\end{aligned}
$$

for a certain coefficient $u_{\pi}>0$ defined in the appendix, where $\pi_{t} \equiv \log \Pi_{t}$ is the inflation rate. Thus we are able to write our stabilization objective purely in terms of the evolution of the aggregate variables $\left\{\hat{Y}_{t}, \pi_{t}\right\}$ and the exogenous disturbances.

We note that when $\Phi>0$, there is a non-zero linear term in (2.2), which means that we cannot expect to evaluate this expression to second order using only an approximate solution for the path of aggregate output that is accurate only to first order. Thus we cannot determine optimal policy, even up to first order, using this approximate objective together with approximations to the structural equations that are accurate only to first order. Rotemberg and Woodford (1997) avoid this problem by assuming an output subsidy (i.e., a value $\bar{\tau}<0$ ) of the size needed to ensure that $\Phi=0$. Here we do not wish to make this assumption, because we assume that lump-sum taxes are unavailable, in which case $\Phi=0$ would be possible only in the case of a particular initial level of government assets $\bar{b}<0$. Furthermore, we are more interested in the case in which government revenue needs are more acute than that would imply.

Benigno and Woodford (2003) propose an alternative way of dealing with this problem, which is to use a second-order approximation to the aggregate-supply relation to eliminate the linear terms in the quadratic welfare measure. In the model that they consider, where taxes are lump-sum (and so do not affect the aggregate supply relation), a forward-integrated second-order approximation to this relation allows one to express the expected discounted value of output terms $\Phi \hat{Y}_{t}$ as a function of purely quadratic terms (except for certain transitory terms that do not affect the "stage two" policy problem). In the present case, the level of distorting taxes has a firstorder effect on the aggregate-supply relation (see equation (2.6) below), so that the forward-integrated relation involves the expected discounted value of the tax rate as 
well as the expected discounted value of output. However, as shown in the appendix, a second-order approximation to the intertemporal solvency condition $(1.15)^{18}$ provides another relation between the expected discounted values of output and the tax rate and a set of purely quadratic terms. These two second-order approximations to the structural equations that appear as constraints in our policy problem can then be used to express the expected discounted value of output terms in (2.2) in terms of purely quadratic terms.

In this manner, we can rewrite $(2.2)$ as

$$
U_{t_{0}}=-\Omega E_{t_{0}} \sum_{t=t_{0}}^{\infty} \beta^{t-t_{0}}\left\{\frac{1}{2} q_{y}\left(\hat{Y}_{t}-\hat{Y}_{t}^{*}\right)^{2}+\frac{1}{2} q_{\pi} \pi_{t}^{2}\right\}+T_{t_{0}}+\text { t.i.p. }+\mathcal{O}\left(\|\xi\|^{3}\right)
$$

where again the coefficients are defined in the appendix. The expression $\widehat{Y}_{t}^{*}$ indicates a function of the vector of exogenous disturbances $\xi_{t}$ defined in the appendix, while $T_{t_{0}}$ is a transitory component. In the case that the alternative policies from date $t_{0}$ onward to be evaluated must be consistent with a vector of prior commitments $X_{t_{0}}$, one can show that the value of the term $T_{t_{0}}$ is implied (to a second-order approximation) by the value of $X_{t_{0}}$. Hence for purposes of characterizing optimal policy from a timeless perspective, it suffices that we rank policies according to the value that they imply for the loss function

$$
E_{t_{0}} \sum_{t=t_{0}}^{\infty} \beta^{t-t_{0}}\left\{\frac{1}{2} q_{y}\left(\hat{Y}_{t}-\hat{Y}_{t}^{*}\right)^{2}+\frac{1}{2} q_{\pi} \pi_{t}^{2}\right\}
$$

where a lower value of (2.4) implies a higher value of (2.3). Because this loss function is purely quadratic (i.e., lacking linear terms), it is possible to evaluate it to second order using only a first-order approximation to the equilibrium evolution of inflation and output under a given policy. Hence log-linear approximations to the structural relations of our model suffice, yielding a standard linear-quadratic policy problem.

In order for this linear-quadratic problem to have a bounded solution (which then approximates the solution to the exact problem), we must verify that the quadratic

\footnotetext{
${ }^{18}$ Since we are interested in providing an approximate characterization of the "stage two" policy problem, in which a precommitted value of $W_{t}$ appears as a constraint, it is actually a second-order approximation to that constraint that we need. However, this latter constraint has the same form as (1.15); the difference is only in which quantities in the relation are taken to have predetermined values.
} 
objective (2.4) is convex. We show in the appendix that $q_{y}, q_{\pi}>0$, so that the objective is convex, as long as the steady-state tax rate $\bar{\tau}$ and share of government purchases $s_{G}$ in the national product are below certain positive bounds. We shall here assume that these conditions are satisfied, i.e., that the government's fiscal needs are not too severe. Note that in this case, our quadratic objective turns out to be of a form commonly assumed in the literature on monetary policy evaluation; that is, policy should seek to minimize the discounted value of a weighted sum of squared deviations of inflation from an optimal level (here zero) and squared fluctuations in an "output gap" $y_{t} \equiv \hat{Y}_{t}-\hat{Y}_{t}^{*}$, where the target output level $\hat{Y}_{t}^{*}$ depends on the various exogenous disturbances in a way discussed in the appendix. It is also perhaps of interest to note that a "tax smoothing" objective of the kind postulated by Barro (1979) and Bohn (1990) does not appear in our welfare measure as a separate objective. Instead, tax distortions are relevant only insofar as they result in "output gaps" of the same sort that monetary stabilization policy aims to minimize.

We turn next to the form of the log-linear constraints in the approximate policy problem. A first-order Taylor series expansion of (1.11) around the zero-inflation steady state yields the log-linear aggregate-supply relation

$$
\pi_{t}=\kappa\left[\hat{Y}_{t}+\psi \hat{\tau}_{t}+c_{\xi}^{\prime} \xi_{t}\right]+\beta E_{t} \pi_{t+1}
$$

for certain coefficients $\kappa, \psi>0$. This is the familiar "New Keynesian Phillips curve" relation, ${ }^{19}$ extended here to take account of the effects of variations in the level of distorting taxes on supply costs.

It is useful to write this approximate aggregate-supply relation in terms of the welfare-relevant output gap $y_{t}$. Equation (2.5) can be equivalently be written as

$$
\pi_{t}=\kappa\left[y_{t}+\psi \hat{\tau}_{t}+u_{t}\right]+\beta E_{t} \pi_{t+1}
$$

where $u_{t}$ is composite "cost-push" disturbance, indicating the degree to which the various exogenous disturbances included in $\xi_{t}$ preclude simultaneous stabilization of inflation, the welfare-relevant output gap, and the tax rate. Alternatively we can write

$$
\pi_{t}=\kappa\left[y_{t}+\psi\left(\hat{\tau}_{t}-\hat{\tau}_{t}^{*}\right)\right]+\beta E_{t} \pi_{t+1}
$$

\footnotetext{
${ }^{19}$ See, e.g., Clarida et al. (1999) or Woodford (2003, chapter 3).
} 
where $\hat{\tau}_{t}^{*} \equiv-\psi^{-1} u_{t}$ indicates the tax change needed at any time to offset the "costpush" shock, in order to allow simultaneous stabilization of inflation and the output gap (the two stabilization objectives reflected in (2.4)).

The effects of the various exogenous disturbances in $\xi_{t}$ on the "cost-push" term $u_{t}$ are explained in the appendix. It is worth noting that under certain conditions $u_{t}$ is unaffected by some disturbances. In the case that $\Phi=0$, the cost-push term is given by

$$
u_{t}=u_{\xi 5} \hat{\mu}_{t}^{w}
$$

where in this case, $u_{\xi 5}=q_{y}^{-1}>0$. Thus the cost-push term is affected only by variations in the wage markup $\hat{\mu}_{t}$; it does not vary in response to taste shocks, technology shocks, government purchases, or variations in government transfers. The reason is that when $\Phi=0$ and neither taxes nor the wage markup vary from their steadystate values, the flexible-price equilibrium is efficient; it follows that level of output consistent with zero inflation is also the one that maximizes welfare, as discussed in Woodford (2003, chapter 6).

Even when $\Phi>0$, if there are no government purchases (so that $s_{G}=0$ ) and no fiscal shocks (meaning that $\hat{G}_{t}=0$ and $\hat{\zeta}_{t}=0$ ), then the $u_{t}$ term is again of the form (2.8), but with $u_{\xi 5}=(1-\Phi) q_{y}^{-1}$, as discussed in Benigno and Woodford (2003). Hence in this case neither taste or technology shocks have "cost-push" effects. The reason is that in this "isoelastic" case, if neither taxes nor the wage markup ever vary, the flexible-price equilibrium value of output and the efficient level vary in exactly the same proportion in response to each of the other types of shocks; hence inflation stabilization also stabilizes the gap between actual output and the efficient level. Another special case is the limiting case of linear utility of consumption $\left(\sigma^{-1}=0\right)$; in this case, $u_{t}$ is again of the form (2.8), for a different value of $u_{\xi 5}$. In general, however, when $\Phi>0$ and $s_{G}>0$, all of the disturbances shift the flexible-price equilibrium level of output (under a constant tax rate) and the efficient level of output to differing extents, resulting in "cost-push" contributions from all of these shocks.

The other constraint on possible equilibrium paths is the intertemporal government solvency condition. A log-linear approximation to (1.15) can be written in the form

$$
\hat{b}_{t-1}-\pi_{t}-\sigma^{-1} y_{t}=-f_{t}+(1-\beta) E_{t} \sum_{T=t}^{\infty} \beta^{T-t}\left[b_{y} y_{T}+b_{\tau}\left(\hat{\tau}_{T}-\hat{\tau}_{T}^{*}\right)\right]
$$


where $\sigma>0$ is the intertemporal elasticity of substitution of private expenditure, and the coefficients $b_{y}, b_{\tau}$ are defined in the appendix, as is $f_{t}$, a composite measure of exogenous "fiscal stress." Here we have written the solvency condition in terms of the same output gap and "tax gap" as equation (2.7), to make clear the extent to which complete stabilization of the variables appearing in the loss function (2.4) is possible. The constraint can also be written in a "flow" form,

$$
\hat{b}_{t-1}-\pi_{t}-\sigma^{-1} y_{t}+f_{t}=(1-\beta)\left[b_{y} y_{t}+b_{\tau}\left(\hat{\tau}_{t}-\hat{\tau}_{t}^{*}\right)\right]+\beta E_{t}\left[\hat{b}_{t}-\pi_{t+1}-\sigma^{-1} y_{t+1}+f_{t+1}\right],
$$

together with a transversality condition. ${ }^{20}$

We note that the only reason why it should not be possible to completely stabilize both inflation and the output gap from some date $t$ onward is if the sum $\hat{b}_{t-1}+f_{t}$ is nonzero. The composite disturbance $f_{t}$ therefore completely summarizes the information at date $t$ about the exogenous disturbances that determines the degree to which stabilization of inflation and output is not possible; and under an optimal policy, the state-contingent evolution of the inflation rate, the output gap, and the real public debt depend solely on the evolution of the single composite disturbance process $\left\{f_{t}\right\}$.

This result contrasts with the standard literature on optimal monetary stabilization policy, in which (in the absence of a motive for interest-rate stabilization, as here) it is instead the cost-push term $u_{t}$ that summarizes the extent to which exogenous disturbances require that fluctuations in inflation and in the output gap should occur. Note that in the case that there are no government purchases and no fiscal shocks, $u_{t}$ corresponds simply to (2.8). Thus, for example, it is concluded (in a model with lump-sum taxes) that there should be no variation in inflation in response to a technology shock (Khan et al., 2002; Benigno and Woodford, 2003). But even in this simple case, the fiscal stress is given by an expression of the form

$$
f_{t} \equiv h_{\xi}^{\prime} \xi_{t}-(1-\beta) E_{t} \sum_{T=t}^{\infty} \beta^{T-t} f_{\xi}^{\prime} \xi_{T},
$$

where the expressions $h_{\xi}^{\prime} \xi_{t}$ and $f_{\xi}^{\prime} \xi_{t}$ both generally include non-zero coefficients on preference and technology shocks, in addition to the markup shock, as shown in the appendix. Hence many disturbances that do not have cost-push effects nonetheless result in optimal variations in both inflation and the output gap.

\footnotetext{
${ }^{20}$ If we restrict attention to bounded paths for the endogenous variables, then a path satisfies $(2.9)$ in each period $t \geq t_{0}$ if and only if it satisfies the flow budget constraint (2.10) in each period.
} 
Finally, we wish to consider optimal policy subject to the constraints that $F_{t_{0}}, K_{t_{0}}$ and $W_{t_{0}}$ take given (precommitted) values. Again, only log-linear approximations to these constraints matter for a log-linear approximate characterization of optimal policy. As discussed in the appendix, the corresponding constraints in our approximate model are precommitments regarding the state-contingent values of $\pi_{t_{0}}$ and $y_{t_{0}}$.

To summarize, our approximate policy problem involves the choice of state-contingent paths for the endogenous variables $\left\{\pi_{t}, y_{t}, \hat{\tau}_{t}, \hat{b}_{t}\right\}$ from some date $t_{0}$ onward so as to minimize the quadratic loss function (2.4), subject to the constraint that conditions (2.7) and (2.9) be satisfied each period, given an initial value $\hat{b}_{t_{0}-1}$ and subject also to the constraints that $\pi_{t_{0}}$ and $y_{t_{0}}$ equal certain precommitted values (that may depend on the state of the world in period $t_{0}$ ). We shall first characterize the state-contingent evolution of the endogenous variables in response to exogenous shocks, in the rationalexpectations equilibrium that solves this problem. We then turn to the derivation of optimal policy rules, commitment to which should implement an equilibrium of this kind.

\section{Optimal Responses to Shocks: The Case of Flex- ible Prices}

In considering the solution to the problem of stabilization policy just posed, it may be useful to first consider the simple case in which prices are fully flexible. This is the limiting case of our model in which $\alpha=0$, with the consequence that $q_{\pi}=0$ in $(2.4)$, and that $\kappa^{-1}=0$ in (2.7). Hence our optimization problem reduces to the minimization of

$$
\frac{1}{2} q_{y} E_{t_{0}} \sum_{t=t_{0}}^{\infty} \beta^{t-t_{0}} y_{t}^{2}
$$

subject to the constraints

$$
y_{t}+\psi\left(\hat{\tau}_{t}-\hat{\tau}_{t}^{*}\right)=0
$$

and (2.9). It is easily seen that in this case, the optimal policy is one that achieves $y_{t}=0$ at all times. Because of $(3.2)$, this requires that $\hat{\tau}_{t}=\hat{\tau}_{t}^{*}$ at all times. The inflation rate is then determined by the requirement of government intertemporal solvency,

$$
\pi_{t}=\hat{b}_{t-1}+f_{t}
$$


This last equation implies that unexpected inflation must equal the innovation in the fiscal stress,

$$
\pi_{t}-E_{t-1} \pi_{t}=f_{t}-E_{t-1} f_{t}
$$

Expected inflation, and hence the evolution of nominal government debt, are indeterminate. If we add to our assumed policy objective a small preference for inflation stabilization, when this has no cost in terms of other objectives, ${ }^{21}$ then the optimal policy will be one that involves $E_{t} \pi_{t+1}=0$ each period, so that the nominal public debt must evolve according to

$$
\hat{b}_{t}=-E_{t} f_{t+1} \text {. }
$$

If, instead, we were to assume the existence of small monetary frictions (and zero interest on money), the tie would be broken by the requirement that the nominal interest rate equal zero each period. ${ }^{22}$ The required expected rate of inflation (and hence the required evolution of the nominal public debt) would then be determined by the variation in the equilibrium real rate of return implied by a real allocation in which $\hat{Y}_{t}=\hat{Y}_{t}^{*}$ each period. That is, one would have $E_{t} \pi_{t+1}=-r_{t}^{*}$, where $r_{t}^{*}$ is the (exogenous) real rate of interest associated output at the target level each period, and so

$$
\hat{b}_{t}=-r_{t}^{*}-E_{t} f_{t+1} \text {. }
$$

We thus obtain simple conclusions about the determinants of fluctuations in inflation, output and the tax rate under optimal policy. Unexpected inflation variations occur as needed in order to prevent taxes from ever having to be varied in order to respond to variations in fiscal stress, as in the analyses of Bohn (1990) and Chari and Kehoe (1999). This allows a model with only riskless nominal government debt

\footnotetext{
${ }^{21}$ Note that this preference can be justified in terms of our model, in the case that $\alpha$ is positive though extremely small. For there will then be a very small positive value for $q_{\pi}$, implying that reduction of the expected discounted value of inflation is preferred to the extent that this does not require any increase in the expected discounted value of squared output gaps.

${ }^{22}$ The result relies upon the fact that the distortions created by the monetary frictions are minimized in the case of a zero opportunity cost of holding money each period, as argued by Friedman (1969). Neither the existence of effects of nominal interest rates on supply costs (so that an interestrate term should appear in the aggregate-supply relation (3.2)) nor the contribution of seignorage revenues to the government budget constraint make any difference to the result, since unexpected changes in revenue needs can always be costlessly obtained through unexpected inflation, while any desired shifts in the aggregate-supply relation to offset cost-push shocks can be achieved by varying the tax rate.
} 
to achieve the same state-contingent allocation of resources as the government would choose to bring about if it were able to issue state-contingent debt, as in the model of Lucas and Stokey (1983).

Because taxes do not have to adjust in response to variations in fiscal stress, as in the tax-smoothing model of Barro (1979), it is possible to "smooth" them across states as well as over time. However, the sense in which it is desirable to "smooth" tax rates is that of minimizing variation in the gap $\hat{\tau}_{t}-\hat{\tau}_{t}^{*}$, rather than variation in the tax rate itself. ${ }^{23}$ In other words, it is really the "tax gap" $\hat{\tau}_{t}-\hat{\tau}_{t}^{*}$ that should be smoothed. Under certain special circumstances, it will not be optimal for tax rates to vary in response to shocks; these are the conditions, discussed above, under which shocks have no cost-push effects, so that there is no change in $\hat{\tau}_{t}^{*}$. For example, if there are no government purchases and there is no variation in the wage markup, this will be the case. But more generally, all disturbances will have some cost-push effect, and result in variations in $\hat{\tau}_{t}^{*}$. There will then be variations in the tax rate in response to these shocks under an optimal policy. However, there will be no unit root in the tax rate, as in the Barro (1979) model of optimal tax policy. Instead, as in the analysis of Lucas and Stokey (1983), the optimal fluctuations in the tax rate will be stationary, and will have the same persistence properties as the real disturbances (specifically, the persistence properties of the composite cost-push shock).

Variations in fiscal stress will instead require changes in the tax rate, as in the analysis of Barro (1979), if we suppose that the government issues only riskless indexed debt, rather than the riskless nominal debt assumed in our baseline model. (Again, for simplicity we assume that only one-period riskless debt is issued.) In this case the objective function (2.4) and the constraints (2.9) and (3.2) remain the same, but $\underline{\mathrm{b}}_{t-1} \equiv \hat{b}_{t-1}-\pi_{t}$, the real value of private claims on the government at the beginning of period $t$, is now a predetermined variable. This means that unexpected inflation variations are no longer able to relax the intertemporal government solvency

${ }^{23}$ A number of authors (e.g., Chari et al., 1991, 1994; Hall and Krieger, 2000; Aiyagari et al., 2002) have found that in calibrated flexible-price models with state-contingent government debt, the optimal variation in labor tax rates is quite small. Our results indicate this as well, in the case that real disturbances have only small cost-push effects, and we have listed earlier various conditions under which this will be the case. But under some circumstances, optimal policy may involve substantial volatility of the tax rate, and indeed, more volatility of the tax rate than of inflation. This would be the case if shocks occur that have large cost-push effects while having relatively little effect on fiscal stress. 
condition. In fact, rewriting the constraint (2.9) in terms of $\underline{b}_{t-1}$, we see that the path of inflation is now completely irrelevant to welfare.

The solution to this optimization problem is now less trivial, as complete stabilization of the output gap is not generally possible. The optimal state-contingent evolution of output and taxes can be determined using a Lagrangian method, as in Woodford (2003, chapter 7). The Lagrangian for the present problem can be written as

$$
\begin{aligned}
\mathcal{L}_{t_{0}}= & E_{t_{0}} \sum_{t=t_{0}}^{\infty} \beta^{t-t_{0}}\left\{\frac{1}{2} q_{y} y_{t}^{2}+\varphi_{1 t}\left[y_{t}+\psi \hat{\tau}_{t}\right]+\varphi_{2 t}\left[\left(\underline{\mathrm{b}}_{t-1}-\sigma^{-1} y_{t}\right)\right.\right. \\
& \left.\left.-(1-\beta)\left(b_{y} y_{t}+b_{\tau} \hat{\tau}_{t}\right)-\beta\left(\underline{\mathrm{b}}_{t}-\sigma^{-1} y_{t+1}\right)\right]\right\}+\sigma \varphi_{2, t_{0}-1} y_{t_{0}},
\end{aligned}
$$

where $\varphi_{1 t}, \varphi_{2 t}$ are Lagrange multipliers associated with constraints (3.2) and (2.10) respectively, ${ }^{24}$ for each $t \geq t_{0}$, and $\sigma \varphi_{2, t_{0}-1}$ is the notation used for the multiplier associated with the additional constraint that $y_{t_{0}}=\bar{y}_{t_{0}}$. The latter constraint is added in order to characterize optimal policy from a timeless perspective, as discussed at the end of section 2; the particular notation used for the multiplier on this constraint results in a time-invariant form for the first-order conditions, as seen below. ${ }^{25} \mathrm{We}$ have dropped terms from the Lagrangian that are not functions of the endogenous variables $y_{t}$ and $\hat{\tau}_{t}$, i.e., products of multipliers and exogenous disturbances, as these do not affect our calculation of the implied first-order conditions.

The resulting first-order condition with respect to $y_{t}$ is

$$
q_{y} y_{t}=-\varphi_{1 t}+\left[(1-\beta) b_{y}+\sigma^{-1}\right] \varphi_{2 t}-\sigma^{-1} \varphi_{2, t-1}
$$

that with respect to $\hat{\tau}_{t}$ is

$$
\psi \varphi_{1 t}=(1-\beta) b_{\tau} \varphi_{2 t}
$$

and that with respect to $\underline{\mathrm{b}}_{t}$ is

$$
\varphi_{2 t}=E_{t} \varphi_{2, t+1}
$$

\footnotetext{
${ }^{24}$ Alternatively, $\varphi_{2 t}$ is the multiplier associated with constraint (2.9).

${ }^{25}$ It should be recalled that in order for policy to be optimal from a timeless perspective, the statecontingent initial commitment $\bar{y}_{t_{0}}$ must be chosen in a way that conforms to the state-contingent commitment regarding $y_{t}$ that will be chosen in all later periods, so that the optimal policy can be implemented by a time-invariant rule. Hence it is convenient to present the first-order conditions in a time-invariant form.
} 
Each of these conditions must be satisfied for each $t \geq t_{0}$, along with the structural equations (3.2) and (2.9) for each $t \geq t_{0}$, for given initial values $\underline{\mathrm{b}}_{t_{0}-1}$ and $y_{t_{0}}$. We look for a bounded solution to these equations, so that (in the event of small enough disturbances) none of the state variables leave a neighborhood of the steady-state values, in which our local approximation to the equilibrium conditions and our welfare objective remain accurate. ${ }^{26}$ Given the existence of such a bounded solution, the transversality condition is necessarily satisfied, so that the solution to these firstorder conditions represents an optimal plan.

An analytical solution to these equations is easily given. Using equation (3.2) to substitute for $\hat{\tau}_{t}$ in the forward-integrated version of (2.9), then equations (3.4) and (3.5) to substitute for $y_{t}$ as a function of the path of $\varphi_{2 t}$, and finally using (3.6) to replace all terms of the form $E_{t} \varphi_{2, t+j}$ (for $j \geq 0$ ) by $\varphi_{2 t}$, we obtain an equation that can be solved for $\varphi_{2 t}$. The solution is of the form

$$
\varphi_{2 t}=\frac{m_{b}}{m_{b}+n_{b}} \varphi_{2, t-1}-\frac{1}{m_{b}+n_{b}}\left[f_{t}+\underline{\mathrm{b}}_{t-1}\right]
$$

coefficients $m_{b}, n_{b}$ are defined in the appendix. The implied dynamics of the government debt are then given by

$$
\underline{\mathrm{b}}_{t}=-E_{t} f_{t+1}-n_{b} \varphi_{2 t}
$$

This allows a complete solution for the evolution of government debt and the multiplier, given the composite exogenous disturbance process $\left\{f_{t}\right\}$, starting from initial conditions $\underline{\mathrm{b}}_{t_{0}-1}$ and $\varphi_{2, t_{0}-1} \cdot{ }^{27}$ Given these solutions, the optimal evolution of the

\footnotetext{
${ }^{26}$ In the only such solution, the variables $\hat{\tau}_{t}, \underline{\mathrm{b}}_{t}$ and $y_{t}$ are all permanently affected by shocks, even when the disturbances are all assumed to be stationary (and bounded) processes. Hence a bounded solution exists only under the assumption that random disturbances occur only in a finite number of periods. However, our characterization of optimal policy does not depend on a particular bound on the number of periods in which there are disturbances, or which periods these are; in order to allow disturbances in a larger number of periods, we must assume a tighter bound on the amplitude of disturbances, in order for the optimal paths of the endogenous variables to remain within a given neighborhood of the steady-state values. Aiyagari et al. (2002) discuss the asymptotic behavior of the optimal plan in the exact nonlinear version of a problem similar to this one, in the case that disturbances occur indefinitely.

${ }^{27}$ The initial condition for $\varphi_{2, t_{0}-1}$ is in turn chosen so that the solution obtained is consistent with the initial constraint $y_{t_{0}}=\bar{y}_{t_{0}}$. Under policy that is optimal from a timeless perspective, this initial commitment is in turn chosen in a self-consistent fashion, as discussed further in section 5 . Note that the specification of $\varphi_{2, t_{0}-1}$ does not affect our conclusions about the optimal responses to shocks, emphasized in this section.
} 
output gap and tax rate are given by

$$
\begin{gathered}
y_{t}=m_{\varphi} \varphi_{2 t}+n_{\varphi} \varphi_{2, t-1}, \\
\hat{\tau}_{t}=\hat{\tau}_{t}^{*}-\psi^{-1} y_{t},
\end{gathered}
$$

where $m_{\varphi}, n_{\varphi}$ are again defined in the appendix. The evolution of inflation remains indeterminate. If we again assume a preference for inflation stabilization when it is costless, optimal policy involves $\pi_{t}=0$ at all times.

In this case, unlike that of nominal debt, inflation is not affected by a pure fiscal shock (or indeed any other shock) under the optimal policy, but instead the output gap and the tax rate are. Note also that in the above solution, the multiplier $\varphi_{2 t}$, the output gap, and the tax rate all follow unit root processes: a temporary disturbance to the fiscal stress permanently changes the level of each of these variables, as in the analysis of the optimal dynamics of the tax rate in Barro (1979) and Bohn (1990). However, the optimal evolution of the tax rate is not in general a pure random walk as in the analysis of Barro and Bohn. Instead, the tax gap is an $\operatorname{IMA}(1,1)$ process, as in the local analysis of Aiyagari et al. (2002); the optimal tax rate $\hat{\tau}_{t}$ may have more complex dynamics, in the case that $\hat{\tau}_{t}^{*}$ exhibits stationary fluctuations. In the special case of linear utility $\left(\sigma^{-1}=0\right), n_{\varphi}=0$, and both the output gap and the tax gap follow random walks (as both co-move with $\varphi_{2 t}$ ). If the only disturbances are

fiscal disturbances $\left(\hat{G}_{t}\right.$ and $\left.\hat{\zeta}_{t}\right)$, then there are also no fluctuations in $\hat{\tau}_{t}^{*}$ in this case, so that the optimal tax rate follows a random walk.

More generally, we observe that optimal policy "smooths" $\varphi_{2 t}$, the value (in units of marginal utility) of additional government revenue in period $t$, so that it follows a random walk. This is the proper generalization of the Barro tax-smoothing result, though it only implies smoothing of tax rates in fairly special cases. We find a similar result in the case that prices are sticky, even when government debt is not indexed, as we now show.

\section{Optimal Responses to Shocks: The Case of Sticky Prices}

We turn now to the characterization of the optimal responses to shocks in the case that prices are sticky $(\alpha>0)$. The optimization problem that provides a first- 
order characterization of optimal responses in this case is that of choosing processes $\left\{\pi_{t}, y_{t}, \hat{\tau}_{t}, \hat{b}_{t}\right\}$ from date $t_{0}$ onward to minimize (2.4), subject to the constraints (2.7) and (2.9) for each $t \geq t_{0}$, together with initial constraints of the form

$$
\pi_{t_{0}}=\bar{\pi}_{t_{0}}, \quad y_{t_{0}}=\bar{y}_{t_{0}}
$$

given the initial condition $\hat{b}_{t_{0}-1}$ and the exogenous evolution of the composite disturbances $\left\{\hat{\tau}_{t}^{*}, f_{t}\right\}$. The Lagrangian for this problem can be written as

$$
\begin{aligned}
\mathcal{L}_{t_{0}}= & E_{t_{0}} \sum_{t=t_{0}}^{\infty} \beta^{t-t_{0}}\left\{\frac{1}{2} q_{y} y_{t}^{2}+\frac{1}{2} q_{\pi} \pi_{t}^{2}+\varphi_{1 t}\left[-\kappa^{-1} \pi_{t}+y_{t}+\psi \hat{\tau}_{t}+\kappa^{-1} \beta \pi_{t+1}\right]+\right. \\
& \left.+\varphi_{2 t}\left[\hat{b}_{t-1}-\pi_{t}-\sigma^{-1} y_{t}-(1-\beta)\left(b_{y} y_{t}+b_{\tau} \hat{\tau}_{t}\right)-\beta\left(\hat{b}_{t}-\pi_{t+1}-\sigma^{-1} y_{t+1}\right)\right]\right\} \\
& +\left[\kappa^{-1} \varphi_{1, t_{0}-1}+\varphi_{2, t_{0}-1}\right] \pi_{t_{0}}+\sigma^{-1} \varphi_{2, t_{0}} y_{t_{0}},
\end{aligned}
$$

by analogy with (3.3).

The first-order condition with respect to $\pi_{t}$ is given by

$$
q_{\pi} \pi_{t}=\kappa^{-1}\left(\varphi_{1 t}-\varphi_{1, t-1}\right)+\left(\varphi_{2 t}-\varphi_{2, t-1}\right)
$$

that with respect to $y_{t}$ is given by

$$
q_{y} y_{t}=-\varphi_{1 t}+\left[(1-\beta) b_{y}+\sigma^{-1}\right] \varphi_{2 t}-\sigma^{-1} \varphi_{2, t-1}
$$

that with respect to $\hat{\tau}_{t}$ is given by

$$
\psi \varphi_{1 t}=(1-\beta) b_{\tau} \varphi_{2 t}
$$

and finally that with respect to $\hat{b}_{t}$ is given by

$$
\varphi_{2 t}=E_{t} \varphi_{2, t+1}
$$

These together with the two structural equations and the initial conditions are to be solved for the state-contingent paths of $\left\{\pi_{t}, \hat{Y}_{t}, \tau_{t}, \hat{b}_{t}, \varphi_{1 t}, \varphi_{2 t}\right\}$. Note that the last three first order conditions are the same as for the flexible-price model with indexed debt; the first condition (4.1) replaces the previous requirement that $\pi_{t}=0$. Hence the solution obtained in the previous section corresponds to a limiting case of this problem, in which $q_{\pi}$ is made unboundedly large; for this reason the discussion above of the more familiar case with flexible prices and riskless indexed government debt provides insight into the character of optimal policy in the present case as well. 
In the unique bounded solution to these equations, the dynamics of government debt and of the shadow value of government revenue $\varphi_{2 t}$ are again of the form

$$
\begin{gathered}
\varphi_{2 t}=\frac{\tilde{m}_{b}}{\tilde{m}_{b}+n_{b}} \varphi_{2, t-1}-\frac{1}{\tilde{m}_{b}+n_{b}}\left[f_{t}+\hat{b}_{t-1}\right], \\
\hat{b}_{t}=-E_{t} f_{t+1}-n_{b} \varphi_{2 t}
\end{gathered}
$$

though the coefficient $\tilde{m}_{b}$ now differs from $m_{b}$, in a way also described in the appendix. The implied dynamics of inflation and output gap are then given by

$$
\begin{aligned}
\pi_{t} & =-\omega_{\varphi}\left(\varphi_{2 t}-\varphi_{2, t-1}\right), \\
y_{t} & =m_{\varphi} \varphi_{2 t}+n_{\varphi} \varphi_{2, t-1},
\end{aligned}
$$

where $m_{\varphi}, n_{\varphi}$ are defined as before, and $\omega_{\varphi}$ is defined in the appendix. The optimal dynamics of the tax rate are those required to make these inflation and outputgap dynamics consistent with the aggregate-supply relation (2.7). Once again, the optimal dynamics of inflation, the output gap, and the public debt depend only on the evolution of the fiscal stress variable $\left\{f_{t}\right\}$; the dynamics of the tax rate also depend on the evolution of $\left\{\hat{\tau}_{t}^{*}\right\}$.

We now discuss the optimal response of the variables to a disturbance to the level of fiscal stress. The laws of motion just derived for government debt and the Lagrange multiplier imply that temporary disturbances to the level of fiscal stress cause a permanent change in the level of both the Lagrange multiplier and the public debt. This then implies a permanent change in the level of output as well, which in turn requires (since inflation is stationary) a permanent change in the level of the tax rate. Since inflation is proportional to the change in the Lagrange multiplier, the price level moves in proportion to the multiplier, which means a temporary disturbance to the fiscal stress results in a permanent change in the price level, as in the flexibleprice case analyzed in the previous section. Thus in this case, the price level, output gap, government debt, and tax rate all have unit roots, combining features of the two special cases considered in the previous section. ${ }^{28}$ Both price level and $\varphi_{2 t}$ are random walks. They jump immediately to new permanent level in response to change in fiscal stress. In the case of purely transitory (white noise) disturbances, government

\footnotetext{
${ }^{28}$ Schmitt-Grohé and Uribe (2001) similarly observe that in a model with sticky prices, the optimal response of the tax rate is similar to what would be optimal in a flexible-price model with riskless indexed government debt.
} 


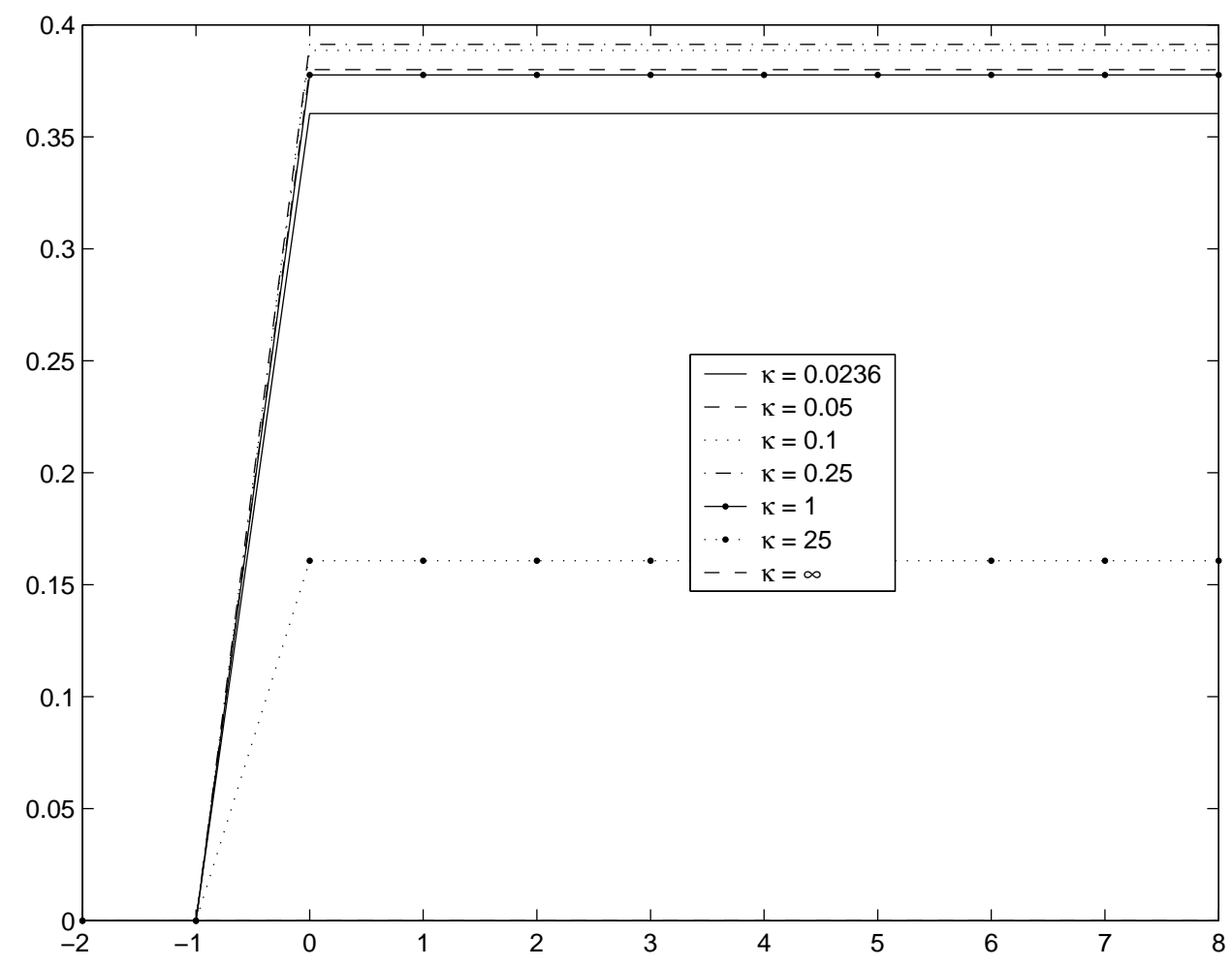

Figure 1: Impulse response of the public debt to a pure fiscal shock, for alternative degrees of price stickiness.

debt also jumps immediately to a new permanent level. Given the dynamics of the price level and government debt, the dynamics of output and tax rate then jointly determined by the aggregate-supply relation and the government budget constraint.

We further find that the degree to which fiscal stress is relieved by a pricelevel jump (as in the flexible-price, nominal-debt case) as opposed to an increase in government debt and hence a permanently higher tax rate (as in the flexibleprice, indexed-debt case) depends on the degree of price stickiness. We illustrate this with a numerical example. We calibrate a quarterly model by assuming that $\beta=0.99, \omega=0.473, \sigma^{-1}=0.157$, and $\kappa=0.0236$, in accordance with the estimates of Rotemberg and Woodford (1997). We furthermore assume an elasticity of substitution among alternative goods of $\theta=10$, an overall level of steady-state distortions $\Phi=1 / 3$, a steady-state tax rate of $\bar{\tau}=0.2$, and a steady-state debt level $\bar{b} / \bar{Y}=2.4$ (debt equal to 60 percent of a year's GDP). Given the assumed degree of market power of producers (a steady-state gross price markup of 1.11) and the assumed size 


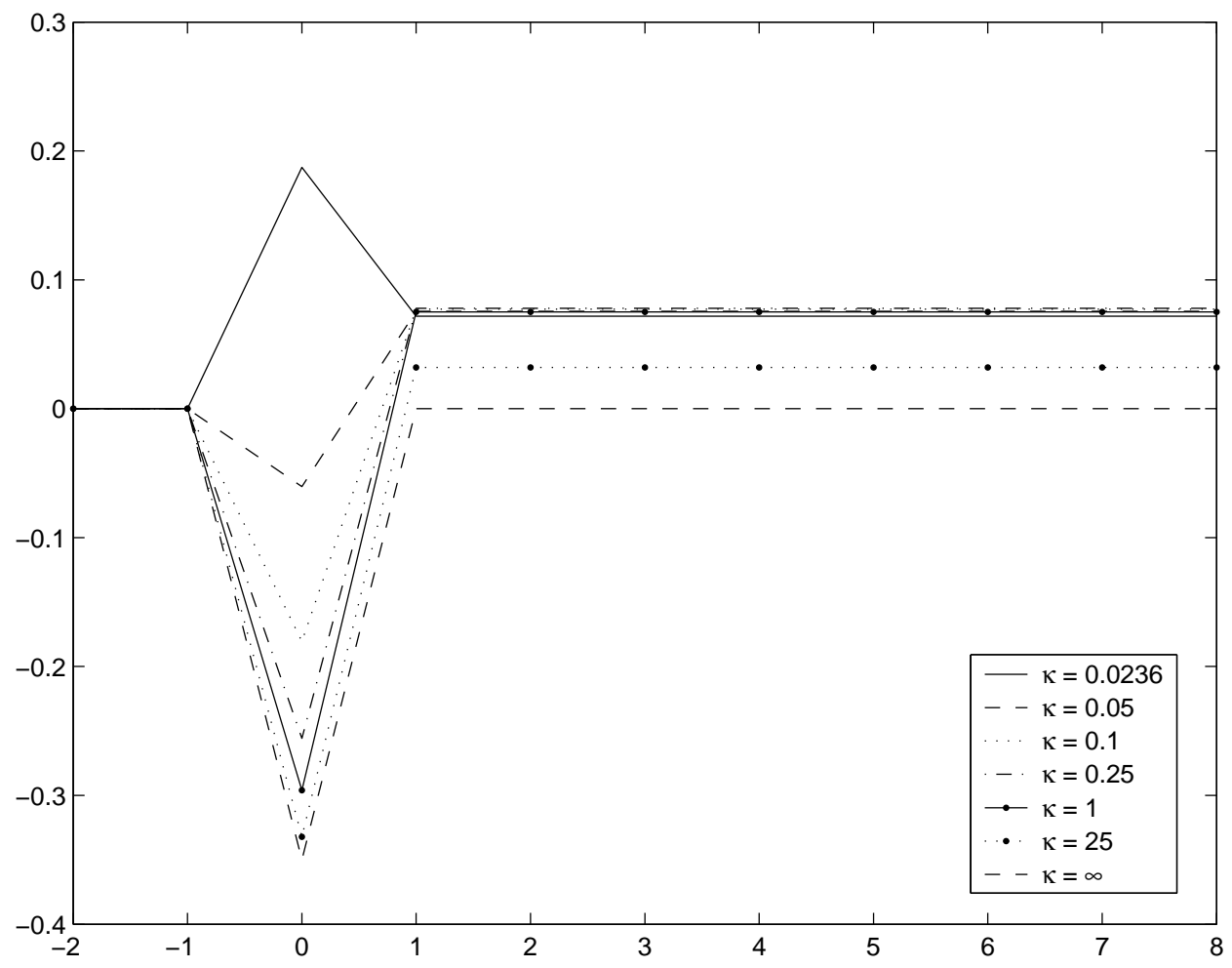

Figure 2: Impulse response of the tax rate to a pure fiscal shock.

of the tax wedge, the value $\Phi=1 / 3$ corresponds to a steady-state wage markup of $\bar{\mu}^{w}=1.08$. If we assume that there are no government transfers in the steady state, then the assumed level of tax revenues net of debt service would finance steady-state government purchases equal to a share $s_{G}=0.176$ of output.

Let us suppose that the economy is disturbed by an exogenous increase in transfer programs $\hat{\zeta}_{t}$, equal to one percent of aggregate output, and expected to last only for the current quarter. Figure 1 shows the optimal impulse response of the government debt $\hat{b}_{t}$ to this shock (where quarter zero is the quarter of the shock), for each of 7 different values for $\kappa$, the slope of the short-run aggregate-supply relation, maintaining the values just stated for the other parameters of the model. The solid line indicates the optimal response in the case of our baseline value for $\kappa$, based on the estimates of Rotemberg and Woodford; the other cases represent progressively greater degrees of price flexibility, up to the limiting case of fully flexible prices (the case $\kappa=\infty$ ). Figures 2 and 3 similarly show the optimal responses of the tax rate and the inflation 


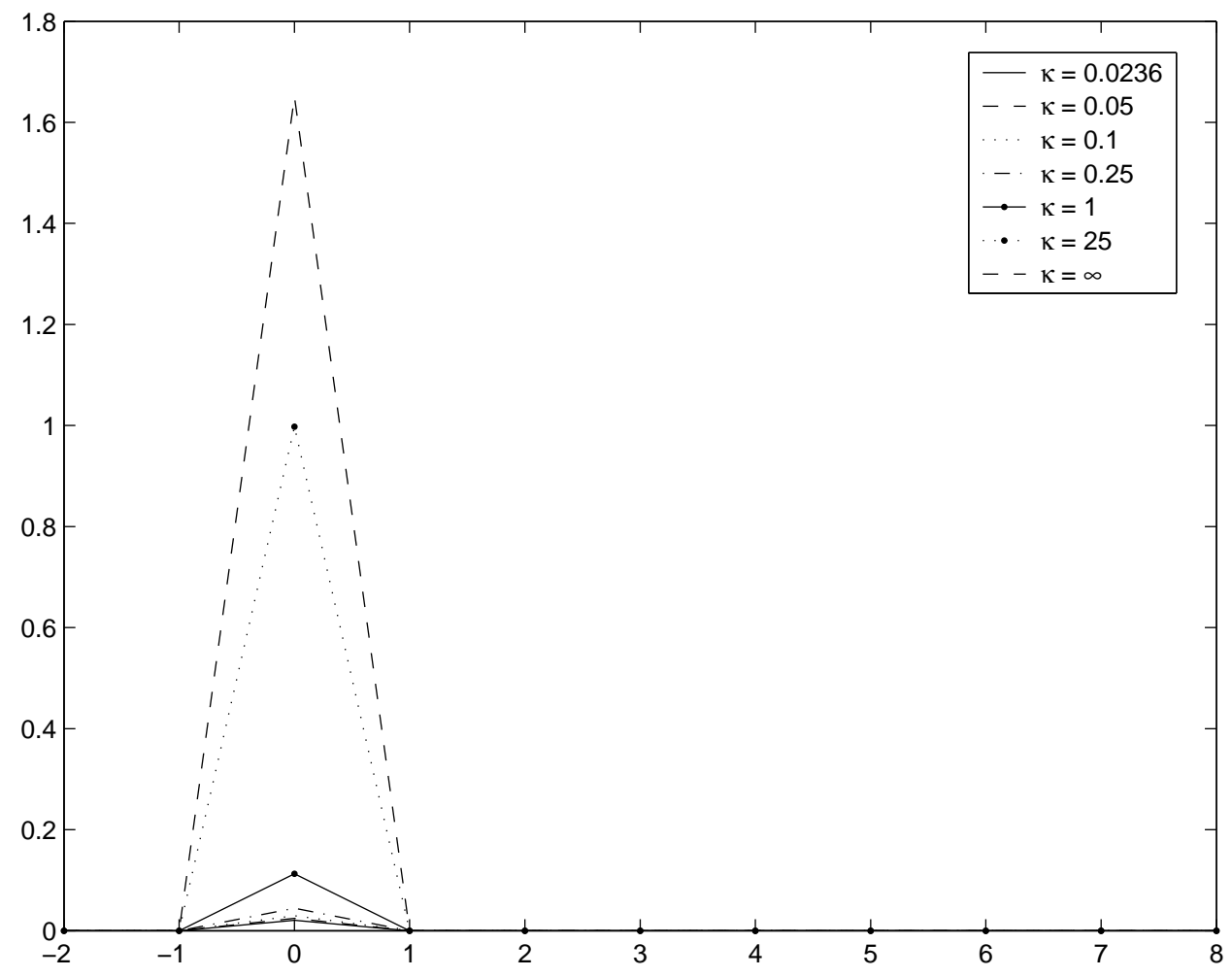

Figure 3: Impulse response of the inflation rate to a pure fiscal shock.

rate to the same disturbance, for each of the same 7 cases. ${ }^{29}$

We see that the volatility of both inflation and tax rates under optimal policy depends greatly on the degree of stickiness of prices. Table 1 reports the initial quarter's response of the inflation rate, and the long-run response of the tax rate, for each of the 7 cases. The table also indicates for each case the implied average time (in weeks) between price changes, $T \equiv(-\log \alpha)^{-1}$, where $0<\alpha<1$ is the fraction of prices unchanged for an entire quarter implied by the assumed value of $\kappa \cdot{ }^{30}$ We

\footnotetext{
${ }^{29}$ In figure 1 , a response of 1 means a one percent increase in the value of $b_{t}$, from 60 percent to 60.6 percent of a year's GDP. In figure 2, a response of 1 means a one percent decrease in $\tau_{t}$, from 20 percent to 20.2 percent. In figure 3 , a response of 1 means a one percent per annum increase in the inflation rate, or an increase of the price level from 1 to 1.0025 over the course of a quarter (given that our model is quarterly). The responses reported in Table 1 are measured in the same way.

${ }^{30}$ We have used the relation between $\alpha$ and $T$ for a continuous-time version of the Calvo model in order to express the degree of price stickiness in terms of an average time between price changes.
} 
Table 1: Immediate responses for alternative degrees of price stickiness.

\begin{tabular}{|cc|cc|}
\hline$\kappa$ & $T$ & $\hat{\tau}_{\infty}$ & $\pi_{0}$ \\
\hline .024 & 29 & .072 & .021 \\
.05 & 20 & .076 & .024 \\
.10 & 14 & .077 & .030 \\
.25 & 9 & .078 & .044 \\
1.0 & 5.4 & .075 & .113 \\
25 & 2.4 & .032 & .998 \\
$\infty$ & 0 & 0 & 1.651 \\
\hline
\end{tabular}

first note that our baseline calibration implies that price changes occur only slightly less frequently than twice per year, which is consistent with survey evidence. ${ }^{31}$ Next, we observe that even were we to assume an aggregate-supply relation several times as steep as the one estimated using U.S. data, our conclusions with regard to the size of the optimal responses of the (long-run) tax rate and the inflation rate would be fairly similar. At the same time, the optimal responses with fully flexible prices are quite different: the response of inflation is 80 times as large as under the baseline sticky-price calibration (implying a variance of inflation 6400 times as large), while the long-run tax rate does not respond at all in the flexible-price case. ${ }^{32}$ But even a small degree of stickiness of prices makes a dramatic difference in the optimal responses; for example, if prices are revised only every five weeks on average, the variance of inflation is reduced by a factor of more than 200, while the optimal response of the long-run tax rate to the increased revenue need is nearly the same size as under the

\footnotetext{
${ }^{31}$ The indicated average time between price changes for the baseline case is shorter than that reported in Rotemberg and Woodford (1997), both because we here assume a slightly larger value of $\theta$, implying a smaller value of $\alpha$, and because of the continuous-time method used here to convert $\alpha$ into an implied average time interval.

${ }^{32}$ The tax rate does respond in the quarter of the shock in the case of flexible prices, but with the opposite sign to that associated with optimal policy under our baseline calibration. Under flexible prices, as discussed above, the tax rate does not respond to variations in fiscal stress at all. Because the increase in government transfers raises the optimal level of output $\hat{Y}_{0}^{*}$, for reasons explained in the appendix, the optimal tax rate $\hat{\tau}_{0}^{*}$ actually falls, in order to induce equilibrium output to increase; and under flexible prices, this is the optimal response of $\hat{\tau}_{0}$.
} 
baseline degree of price stickiness. Thus we find, as do Schmitt-Grohé and Uribe (2001) in the context of a calibrated model with convex costs of price adjustment, that the conclusions of the flexible-price analysis are quite misleading if prices are even slightly sticky. Under a realistic calibration of the degree of price stickiness, inflation should be quite stable, even in response to disturbances with substantial consequences for the government's budget constraint, while tax rates should instead respond substantially (and with a unit root) to variations in fiscal stress.

We can also compare our results with those that arise when taxes are lump-sum. In this case, $\psi=0$, and the first-order condition (4.3) requires that $\varphi_{2 t}=0$. The remaining first-order conditions reduce to

$$
\begin{gathered}
q_{\pi} \pi_{t}=\kappa^{-1}\left(\varphi_{1 t}-\varphi_{1, t-1}\right) \\
q_{y} y_{t}=-\varphi_{1 t}
\end{gathered}
$$

for each $t \geq t_{0}$ as in Clarida et al. (1999) and Woodford (2003, chapter 7). In this case the fiscal stress is no longer relevant for inflation or output-gap determination. Instead, only the cost-push shock $u_{t}$ is responsible for incomplete stabilization. The determinants of the cost-push effects of underlying disturbances, and of the target output level $\hat{Y}_{t}^{*}$ are also somewhat different, because in this case $\vartheta_{1}=0$. For example, a pure fiscal shock has no cost-push effect, nor any effect on $\hat{Y}_{t}^{*}$, and hence no effect on the optimal evolution of either inflation or output. ${ }^{33}$ Furthermore, as shown in the references just mentioned, the price level no longer follows a random walk; instead, it is a stationary variable. Increases in the price level due to a cost-push shock are subsequently undone by period of deflation.

Note that the familiar case from the literature on monetary stabilization policy does not result simply from assuming that sources of revenue that do not shift the aggregate-supply relation are available; it is also important that the sort of tax that does shift the AS relation (like the sales tax here) is not available. We could nest both the standard model and our present baseline case within a single, more general framework by assuming that revenue can be raised using either the sales tax or a lumpsum tax, but that there is an additional convex cost (perhaps representing "collection costs", assumed to reduce the utility of the representative household but not using

\footnotetext{
${ }^{33}$ See Benigno and Woodford (2003) for detailed analysis of the determinants of $u_{t}$ and $\hat{Y}_{t}^{*}$ in this case.
} 
real resources) of increases in either tax rate. The standard case would then appear as the limiting case of this model in which the collection costs associated with the sales tax are infinite, while those associated with the lump-sum tax are zero; the baseline model here would correspond to an alternative limiting case in which the collection costs associated with the lump-sum tax are infinite, while those associated with the sales tax are zero. In intermediate cases, we would continue to find that fiscal stress affects the optimal evolution of both inflation and the output gap, as long as there is a positive collection cost for the lump-sum tax. At the same time, the result that the shadow value of additional government revenue follows a random walk under optimal policy (which would still be true) will not in general imply, as it does here, that the price level should also be a random walk; for the perfect co-movement of $\varphi_{1 t}$ and $\varphi_{2 t}$ that characterizes optimal policy in our baseline case will not be implied by the first-order conditions except in the case that there are no collection costs associated with the sales tax. Nonetheless, the price level will generally contain a unit root under optimal policy, even if it will not generally follow a random walk.

We also obtain results more similar to those in the standard literature on monetary stabilization policy if we assume (realistically) that it is not possible to adjust tax rates on such short notice in response to shocks as is possible with monetary policy. As a simple way of introducing delays in the adjustment of tax policy, suppose that the tax rate $\tau_{t}$ has to be fixed in period $t-d$. In this case, the first-order conditions characterizing optimal responses to shocks are the same as above, except that (4.3) is replaced by

$$
\psi E_{t} \varphi_{1, t+d}=(1-\beta) b_{\tau} E_{t} \varphi_{2, t+d}
$$

for each $t \geq t_{0}$. In this case, the first-order conditions imply that $E_{t} \pi_{t+d+1}=0$, but no longer imply that changes in the price level must be unforecastable from one period to the next. As a result, price-level increases in response to disturbances are typically partially, but not completely, undone in subsequent periods. Yet there continues to be a unit root in the price level (of at least a small innovation variance), even in the case of an arbitrarily long delay $d$ in the adjustment of tax rates. 


\section{Optimal Targeting Rules for Monetary and Fis- cal Policy}

We now wish to characterize the policy rules that the monetary and fiscal authorities can follow in order to bring about the state-contingent responses to shocks described in the previous section. One might think that it suffices to solve for the optimal statecontingent paths for the policy instruments. But in general this is not a desirable approach to the specification of a policy rule, as discussed in Svensson (2003) and Woodford (2003, chapter 7). A description of optimal policy in these terms would require enumeration of all of the types of shocks that might be encountered later, indefinitely far in the future, which is not feasible in practice. A commitment to a state-contingent instrument path, even when possible, also may not determine the optimal equilibrium as the locally unique rational-expectations equilibrium consistent with this policy; many other (much less desirable) equilibria may also be consistent with the same state-contingent instrument path.

Instead, we here specify targeting rules in the sense of Svensson (1999, 2003) and Giannoni and Woodford (2003). These targeting rules are commitments on the part of the policy authorities to adjust their respective instruments so as to ensure that the projected paths of the endogenous variables satisfy certain target criteria. We show that under an appropriate choice of these target criteria, a commitment to ensure that they hold at all times will determine a unique non-explosive rationalexpectations equilibrium, in which the state-contingent evolution of inflation, output and the tax rate solves the optimization problem discussed in the previous section. Moreover, we show that it is possible to obtain a specification of the policy rules that is robust to alternative specifications of the exogenous shock processes.

We apply the general approach of Giannoni and Woodford (2002), which allows the derivation of optimal target criteria with the properties just stated. In addition, Giannoni and Woodford show that such target criteria can be formulated that refer only to the projected paths of the target variables (the ones in terms of which the stabilization objectives of policy are defined - here, inflation and the output gap). Briefly, the method involves constructing the target criteria by eliminating

the Lagrange multipliers from the system of the system of first-order conditions that characterize the optimal state-contingent evolution, regardless of character of the (additive) disturbances. We are left with linear relations among the target variables, that 
do not involve the disturbances and with coefficients independent of the specification of the disturbances, that represent the desired target criteria.

Recall that the first-order conditions that characterize the optimal state-contingent paths in the problem considered in the previous section are given by (4.1) - (4.4). As explained in the previous section, the first three of these conditions imply that the evolution of inflation and of the output gap must satisfy (4.5) - (4.6) each period. We can solve $(4.5)$ - (4.6) for the values of $\varphi_{2 t}, \varphi_{2, t-1}$ implied by the values of $\pi_{t}, y_{t}$ that are observed in an optimal equilibrium. We can then replace $\varphi_{2, t-1}$ in these two relations by the multiplier implied in this way by observed values of $\pi_{t-1}, y_{t-1}$. Finally, we can eliminate $\varphi_{2 t}$ from these two relations, to obtain a necessary relation between $\pi_{t}$ and $y_{t}$, given $\pi_{t-1}$ and $y_{t-1}$, given by

$$
\pi_{t}+\frac{n_{\varphi}}{m_{\varphi}} \pi_{t-1}+\frac{\omega_{\varphi}}{m_{\varphi}}\left(y_{t}-y_{t-1}\right)=0
$$

This target criterion has the form of a "flexible inflation target," similar to the optimal target criterion for monetary policy in model with lump-sum taxation (Woodford, 2003, chapter 7). It is interesting to note that, as in all of the examples of optimal target criteria for monetary policy derived under varying assumptions in Giannoni and Woodford (2003), it is only the projected rate of change of the output gap that matters for determining the appropriate adjustment of the near-term inflation target; the absolute level of the output gap is irrelevant.

The remaining first-order condition from the previous section, not used in the derivation of (5.1), is (4.4). By similarly using the solutions for $\varphi_{2, t+1}, \varphi_{2 t}$ implied by observations of $\pi_{t+1}, y_{t+1}$ to substitute for the multipliers in this condition, one obtains a further target criterion

$$
E_{t} \pi_{t+1}=0
$$

(Note that the fact that this always holds in the optimal equilibrium - i.e., that the price level must follow a random walk — has already been noted in the previous section.) We show in the appendix that policies that ensure that (5.1) - (5.2) hold for all $t \geq t_{0}$ determine a unique non-explosive rational-expectations equilibrium.

Moreover, this equilibrium solves the above first-order conditions for a particular specification of the initial lagged multipliers $\varphi_{1, t_{0}-1}, \varphi_{2, t_{0}-1}$, which are inferred from the initial values $\pi_{t_{0}-1}, y_{t_{0}-1}$ in the way just explained. Hence this equilibrium minimizes expected discounted losses (2.4) given $\hat{b}_{t_{0}-1}$ and subject to constraints on initial 
outcomes of the form

$$
\begin{aligned}
& \pi_{t_{0}}=\bar{\pi}\left(\pi_{t_{0}-1}, y_{t_{0}-1}\right), \\
& y_{t_{0}}=\bar{y}\left(\pi_{t_{0}-1}, y_{t_{0}-1}\right) .
\end{aligned}
$$

Furthermore, these constraints are self-consistent in the sense that the equilibrium that solves this problem is one in which $\pi_{t}, y_{t}$ are chosen to satisfy equations of this form in all periods $t>t_{0}$. Hence these time-invariant policy rules are optimal from a timeless perspective. ${ }^{34}$ And they are optimal regardless of the specification of disturbance processes. Thus we have obtained robustly optimal target criteria, as desired.

We have established a pair of target criteria with the property that if they are expected to be jointly satisfied each period, the resulting equilibrium involves the optimal responses to shocks. This result in itself, however, does not establish which policy instrument should be used to ensure satisfaction of which criterion. Since the variables referred to in both criteria can be affected by both monetary and fiscal policy, there is not a uniquely appropriate answer to that question. However, the following represents a relatively simple example of a way in which such a regime could be institutionalized through separate targeting procedures on the part of monetary and fiscal authorities.

Let the central bank be assigned the task of maximizing social welfare through its adjustment of the level of short-term interest rates, taking as given the statecontingent evolution of the public debt $\left\{\hat{b}_{t}\right\}$, which depends on the decisions of the fiscal authority. Thus the central bank treats the evolution of the public debt as being outside its control, just like the exogenous disturbances $\left\{\xi_{t}\right\}$, and simply seeks to forecast its evolution in order to correctly model the constraints on its own policy. Here we do not propose a regime under which it is actually true that the evolution of the public debt would be unaffected by a change in monetary policy. But there is no inconsistency in the central bank's assumption (since a given bounded process $\left\{\hat{b}_{t}\right\}$ will continue to represent a feasible fiscal policy regardless of the policy adopted by the central bank), and we shall show that the conduct of policy under this assumption does not lead to a suboptimal outcome, as long as the state-contingent evolution of the public debt is correctly forecasted by the central bank.

\footnotetext{
${ }^{34}$ See Woodford $(2003$, chapters 7,8$)$ for further discussion of the self-consistency condition that the initial constraints are required to satisfy.
} 
The central bank then seeks to bring about paths for $\left\{\pi_{t}, y_{t}, \hat{\tau}_{t}\right\}$ from date $t_{0}$ onward that minimize (2.4), subject to the constraints (2.7) and (2.9) for each $t \geq t_{0}$, together with initial constraints of the form (5.3) - (5.4), given the evolution of the processes $\left\{\hat{\tau}_{t}^{*}, f_{t}, \hat{b}_{t}\right\}$. The first-order conditions for this optimization problem are given by (4.1), (4.2) and (4.4) each period, which in turn imply that (5.1) must hold each period, as shown above. One can further show that a commitment by the central bank to ensure that (5.1) holds each period determines the equilibrium evolution that solves this problem, in the case of an appropriate (self-consistent) choice of the initial constraints (5.3) - (5.4). Thus (5.1) is an optimal target criterion for a policy authority seeking to solve the kind of problem just posed; and since the problem takes as given the evolution of the public debt, it is obviously a more suitable assignment for the central bank than for the fiscal authority. The kind of interest-rate reaction function that can be used to implement a "flexible inflation target" of this kind is discussed in Svensson and Woodford (2003) and Woodford (2003, chapter 7).

Correspondingly, let the fiscal authority be assigned the task of choosing the level of government revenue each period that will maximize social welfare, taking as given the state-contingent evolution of output $\left\{y_{t}\right\}$, which it regards as being determined by monetary policy. (Again, it need not really be the case that the central bank ensures a particular state-contingent path of output, regardless of what the fiscal authority does. But again, this assumption is not inconsistent with our model of the economy, since it is possible for the central bank to bring about any bounded process $\left\{y_{t}\right\}$ that it wishes, regardless of fiscal policy, in the case that prices are sticky.) If the fiscal authority regards the evolution of output as outside its control, its objective reduces to the minimization of

$$
E_{t_{0}} \sum_{t=t_{0}}^{\infty} \beta^{t-t_{0}} \pi_{t}^{2}
$$

But this is a possible objective for fiscal policy, given the effects of tax policy on inflation dynamics (when taxes are not lump-sum) indicated by (2.7).

Forward integration of (2.7) implies that

$$
\pi_{t}=\kappa E_{t} \sum_{T=t}^{\infty} \beta^{T-t} y_{T}+\kappa \psi E_{t} \sum_{T=t}^{\infty} \beta^{T-t}\left(\hat{\tau}_{T}-\hat{\tau}_{T}^{*}\right) .
$$

Thus what matters about fiscal policy for current inflation determination is the present value of expected tax rates; but this in turn is constrained by the intertemporal solvency condition (2.9). Using (2.9) to substitute for the present value of taxes 
in (5.6), we obtain a relation of the form

$$
\pi_{t}=\mu_{1}\left[\hat{b}_{t-1}-\sigma^{-1} y_{t}+f_{t}\right]+\mu_{2} E_{t} \sum_{T=t}^{\infty} \beta^{T-t} y_{T}
$$

for certain coefficients $\mu_{1}, \mu_{2}>0$ defined in the appendix. If the fiscal authority takes the evolution of output as given, then this relation implies that its policy in period $t$ can have no effect on $\pi_{t}$. However, it can affect inflation in the following

period through the effects the current government budget on $\hat{b}_{t}$. Furthermore, since the choice of $\hat{b}_{t}$ has no effect on inflation in later periods (given that it places no constraint on the level of public debt that may be chosen in later periods), $\hat{b}_{t}$ should be chosen so as to minimize $E_{t} \pi_{t+1}^{2}$.

The first-order condition for the optimal choice of $\hat{b}_{t}$ is then simply (5.2), which we find is indeed a suitable target criterion for the fiscal authority. The decision rule implied by this target criterion is seen to be

$$
\hat{b}_{t}=-E_{t} f_{t+1}+\sigma^{-1} E_{t} y_{t+1}-\left(\mu_{2} / \mu_{1}\right) E_{t} \sum_{T=t+1}^{\infty} \beta^{T-t-1} y_{T}
$$

which expresses the optimal level of government borrowing as a function of the fiscal authority's projections of the exogenous determinants of fiscal stress and of future real activity. It is clearly possible for the fiscal authority to implement this target criterion, and doing so leads to a determinate equilibrium path for inflation, given the path of output. We thus obtain a pair of targeting rules, one for the central bank and one for the fiscal authority, that if both pursued will implement an equilibrium that is optimal from a timeless perspective. Furthermore, each individual rule can be rationalized as a solution to a constrained optimization problem that the particular policy authority is assigned to solve.

\section{Conclusion}

We have shown that it is possible to jointly analyze optimal monetary and fiscal policy within a single framework. The two problems, often considered in isolation, turn out to be more closely related than might have been expected. In particular, we find that variations in the level of distorting taxes should be chosen to serve the same objectives as those emphasized in the literature on monetary stabilization policy: stabilization 
of inflation and of a (properly defined) output gap. A single output gap can be defined that measures the total distortion of the level of economic activity, resulting both from the stickiness of prices (and the consequent variation in markups) and from the supply-side effects of tax distortions. It is this cumulative gap that one wishes to stabilize, rather than the individual components resulting from the two sources; and both monetary policy and tax policy can be used to affect it. Both monetary policy and tax policy also matter for inflation determination in our model, because of the effects of the tax rate on real marginal cost and hence on the aggregate-supply relation. Indeed, we have exhibited a pair of robustly optimal targeting rules for the monetary and fiscal authorities respectively, under which both authorities consider the consequences of their actions for near-term inflation projections in determining how to adjust their instruments.

And not only should the fiscal authority use tax policy to serve the traditional goals of monetary stabilization policy; we also find that the monetary authority should take account of the consequences of its actions for the government budget. In the present model, that abstracts entirely from transactions frictions, these consequences have solely to do with the implications of alternative price-level and interest-rate paths for the real burden of interest payments on the public debt, and not any contribution of seignorage to government revenues. Nonetheless, under a calibration of our model that assumes a debt burden and a level of distorting taxes that would not be unusual for an advanced industrial economy, taking account of the existence of a positive shadow value of additional government revenue (owing to the non-existence of lumpsum taxes) makes a material difference for the quantitative characterization of optimal monetary policy. In fact, we have found that the crucial summary statistic that indicates the degree to which various types of real disturbances should be allowed to affect short-run projections for either inflation or the output gap is not the degree to which these disturbances shift the aggregate-supply curve for a given tax rate (i.e., the extent to which they represent "cost-push" shocks), but rather the degree to which they create fiscal stress (shift the intertemporal government solvency condition).

Our conclusion that monetary policy should take account of the requirements for government solvency does not imply anything as strong as the result of Chari and Kehoe (1999) for a flexible-price economy with nominal government debt, according to which surprise variations in the inflation rate should be used to completely offset variations in fiscal stress, so that tax rates need not vary (other than as necessary to 
stabilize the output gap). We find that in the case of even a modest degree of price stickiness - much less than what seems to be consistent with empirical evidence for the U.S. - it is not optimal for inflation to respond to variations in fiscal stress by more than a tiny fraction of the amount that would be required to eliminate the fiscal stress (and that would be optimal with fully flexible prices); instead, a substantial part of the adjustment should come through a change in the tax rate. But the way in which the acceptable short-run inflation projection should be affected by variations in the projected output gap is substantially different in an economy with only distorting taxes than would be the case in the presence of lump-sum taxation. For with distorting taxes, the available tradeoff between variations in inflation and in the output gap depends not only on the way these variables are related to one another through the aggregate-supply relation, but also on the way that each of them affects the government budget. 


\section{A Appendix}

\section{A.1 Derivation of the aggregate-supply relation (equation (1.11))}

In this section, we derive equation (1.11) in the main text and we define the variables $F_{t}$ and $K_{t}$. In the Calvo model, a supplier that changes its price in period $t$ chooses a new price $p_{t}(i)$ to maximize

$$
E_{t}\left\{\sum_{T=t}^{\infty} \alpha^{T-t} Q_{t, T} \Pi\left(p_{t}(i), p_{T}^{j}, P_{T} ; Y_{T}, \tau_{T}, \xi_{T}\right)\right\}
$$

where $\alpha^{T-t}$ is the probability that the price set at time $t$ remains fixed in period $T$, $Q_{t, T}$ is the stochastic discount factor given by $(1.7)$, and the profit function $\Pi(\cdot)$ is defined as

$\Pi\left(p, p^{j}, P ; Y, \tau, \xi\right) \equiv(1-\tau) p Y(p / P)^{-\theta}-\mu^{w} \frac{\tilde{v}_{h}\left(f^{-1}\left(Y\left(p^{j} / P\right)^{-\theta} / A\right) ; \xi\right)}{\tilde{u}_{c}(Y-G ; \xi)} P \cdot f^{-1}\left(Y(p / P)^{-\theta} / A\right)$.

Here Dixit-Stiglitz monopolistic competition implies that the individual supplier faces a demand curve each period of the form

$$
y_{t}(i)=Y_{t}\left(p_{t}(i) / P_{t}\right)^{-\theta}
$$

so that after-tax sales revenues are the function of $p$ given in the first term on the right-hand side of (A.8). The second term indicates the nominal wage bill, obtained by inverting the production function to obtain the required labor input, and multiplying this by the industry wage for sector $j$. The industry wage is obtained from the labor supply equation (1.8), under the assumption that each of the firms in industry $j$ (other than $i$, assumed to have a negligible effect on industry labor demand) charges the common price $p^{j}$. (Because all firms in a given industry are assumed to adjust their prices at the same time, in equilibrium the prices of firms in a given industry are always identical. We must nonetheless define the profit function for the case in which firm $i$ deviates from the industry price, in order to determine whether the industry price is optimal for each individual firm.)

We note that supplier $i$ 's profits are a concave function of the quantity sold $y_{t}(i)$, since revenues are proportional to $y_{t}^{\frac{\theta-1}{\theta}}(i)$ and hence concave in $y_{t}(i)$, while costs are 
convex in $y_{t}(i)$. Moreover, since $y_{t}(i)$ is proportional to $p_{t}(i)^{-\theta}$, the profit function is also concave in $p_{t}(i)^{-\theta}$. The first-order condition for the optimal choice of the price $p_{t}(i)$ is the same as the one with respect to $p_{t}(i)^{-\theta}$; hence the first-order condition with respect to $p_{t}(i)$ is both necessary and sufficient for an optimum.

For this first-order condition, we obtain

$$
E_{t}\left\{\sum_{T=t}^{\infty} \alpha^{T-t} Q_{t, T}\left(\frac{p_{t}(i)}{P_{T}}\right)^{-\theta} Y_{T} \Psi_{T}\left(p_{t}(i), p_{t}^{j}\right)\right\}=0
$$

with

$$
\Psi_{T}\left(p, p^{j}\right) \equiv\left[\left(1-\tau_{T}\right)-\frac{\theta}{\theta-1} \mu_{T}^{w} \frac{\tilde{v}_{h}\left(f^{-1}\left(Y_{T}\left(p^{j} / P_{T}\right)^{-\theta} / A_{T}\right) ; \xi_{T}\right)}{\tilde{u}_{c}\left(Y_{T}-G_{T} ; \xi_{T}\right) \cdot A_{T} f^{\prime}\left(f^{-1}\left(Y_{T}\left(p / P_{T}\right)^{-\theta} / A_{T}\right)\right)} \frac{P_{T}}{p}\right]
$$

Using the definitions

$$
\begin{gathered}
u\left(Y_{t} ; \xi_{t}\right) \equiv \tilde{u}\left(Y_{t}-G_{t} ; \xi_{t}\right), \\
v\left(y_{t}(i) ; \xi_{t}\right) \equiv \tilde{v}\left(f^{-1}\left(y_{t}(i) / A_{t}\right) ; \xi_{t}\right)=\tilde{v}\left(H_{t}(i) ; \xi_{t}\right),
\end{gathered}
$$

and noting that each firm in an industry will set the same price, so that $p_{t}(i)=p_{t}^{j}=$ $p_{t}^{*}$, the common price of all goods with prices revised at date $t$, we can rewrite the above first-order condition as

$$
E_{t}\left\{\sum_{T=t}^{\infty}(\alpha \beta)^{T-t} Q_{t, T}\left(\frac{p_{t}^{*}}{P_{T}}\right)^{-\theta} Y_{T}\left[\left(1-\tau_{T}\right)-\frac{\theta}{\theta-1} \mu_{T}^{w} \frac{v_{y}\left(Y_{T}\left(p_{t}^{*} / P_{T}\right)^{-\theta} ; \xi_{T}\right)}{u_{c}\left(Y_{T} ; \xi_{T}\right)} \frac{P_{T}}{p_{t}^{*}}\right]\right\}=0 .
$$

Substituting the equilibrium value for the discount factor, we finally obtain

$$
E_{t}\left\{\sum_{T=t}^{\infty} \alpha^{T-t} u_{c}\left(Y_{T} ; \xi_{T}\right)\left(\frac{p_{t}^{*}}{P_{T}}\right)^{-\theta} Y_{T}\left[\frac{p_{t}^{*}}{P_{T}}\left(1-\tau_{T}\right)-\frac{\theta}{\theta-1} \mu_{T}^{w} \frac{v_{y}\left(Y_{T}\left(p_{t}^{*} / P_{T}\right)^{-\theta} ; \xi_{T}\right)}{u_{c}\left(Y_{T} ; \xi_{T}\right)}\right]\right\}=0 .
$$

Using the isoelastic functional forms given in the text, we obtain a closed-form solution to (A.9), given by

$$
\frac{p_{t}^{*}}{P_{t}}=\left(\frac{K_{t}}{F_{t}}\right)^{\frac{1}{1+\omega \theta}}
$$

where $F_{t}$ and $K_{t}$ are aggregate variables of the form

$$
F_{t} \equiv E_{t} \sum_{T=t}^{\infty}(\alpha \beta)^{T-t}\left(1-\tau_{T}\right) f\left(Y_{T} ; \xi_{T}\right)\left(\frac{P_{T}}{P_{t}}\right)^{\theta-1}
$$




$$
K_{t} \equiv E_{t} \sum_{T=t}^{\infty}(\alpha \beta)^{T-t} k\left(Y_{T} ; \xi_{T}\right)\left(\frac{P_{T}}{P_{t}}\right)^{\theta(1+\omega)},
$$

in which expressions

$$
\begin{gathered}
f(Y ; \xi) \equiv u_{c}(Y ; \xi) Y, \\
k(Y ; \xi) \equiv \frac{\theta}{\theta-1} \mu^{w} v_{y}(Y ; \xi) Y,
\end{gathered}
$$

and where in the function $k(\cdot)$, the vector of shocks has been extended to include the shock $\mu_{t}^{w}$. Substitution of (A.10) into the law of motion for the Dixit-Stiglitz price index

$$
P_{t}=\left[(1-\alpha) p_{t}^{* 1-\theta}+\alpha P_{t-1}^{1-\theta}\right]^{\frac{1}{1-\theta}}
$$

yields a short-run aggregate-supply relation between inflation and output of the form (1.11) in the text.

\section{A.2 Recursive formulation of the policy problem}

Under the standard (Ramsey) approach to the characterization of an optimal policy commitment, one chooses among state-contingent paths $\left\{\Pi_{t}, Y_{t}, \tau_{t}, b_{t}, \Delta_{t}\right\}$ from some initial date $t_{0}$ onward that satisfy

$$
\begin{gathered}
\frac{1-\alpha \Pi_{t}^{\theta-1}}{1-\alpha}=\left(\frac{F_{t}}{K_{t}}\right)^{\frac{\theta-1}{1+\omega \theta}}, \\
\Delta_{t}=h\left(\Delta_{t-1}, \Pi_{t}\right), \\
b_{t-1} \frac{P_{t-1}}{P_{t}}=E_{t} \sum_{T=t}^{\infty} R_{t, T} s_{T},
\end{gathered}
$$

where

$$
s_{t} \equiv \tau_{t} Y_{t}-G_{t}-\zeta_{t}
$$

for each $t \geq t_{0}$, given initial government debt $b_{t_{0}-1}$ and price dispersion $\Delta_{t_{0}-1}$, so as to maximize

$$
U_{t_{0}}=E_{t_{0}} \sum_{t=t_{0}}^{\infty} \beta^{t-t_{0}} U\left(Y_{t}, \Delta_{t} ; \xi_{t}\right) .
$$

Here we note that the definition (1.3) of the index of price dispersion implies the law of motion

$$
\Delta_{t}=\alpha \Delta_{t-1} \Pi_{t}^{\theta(1+\omega)}+(1-\alpha)\left(\frac{1-\alpha \Pi_{t}^{\theta-1}}{1-\alpha}\right)^{-\frac{\theta(1+\omega)}{1-\theta}}
$$


which can be written in the form (A.17); this is the origin of that constraint.

We now show that the $t_{0}$-optimal plan (Ramsey problem) can be obtained as the solution to a two-stage optimization problem. To this purpose, let

$$
W_{t} \equiv E_{t} \sum_{T=t}^{\infty} \beta^{T-t} \tilde{u}_{c}\left(Y_{T}-G_{T} ; \xi_{T}\right) s_{T},
$$

and let $\mathcal{F}$ be the set of values for $\left(b_{t-1}, \Delta_{t-1}, F_{t}, K_{t}, W_{t}\right)$ such that there exist paths $\left\{\Pi_{T}, Y_{T}, \tau_{T}, b_{T}, \Delta_{T}\right\}$ for dates $T \geq t$ that satisfy (A.16), (A.17) and (A.18) for each $T$, that are consistent with the specified values for $F_{t}, K_{t}$, defined in (A.23) and (A.24), and $W_{t}$, and that imply a well-defined value for the objective $U_{t}$ defined in (A.20). Furthermore, for any $\left(b_{t-1}, \Delta_{t-1}, F_{t}, K_{t}, W_{t}\right) \in \mathcal{F}$, let $V\left(b_{t-1}, \Delta_{t-1}, X_{t} ; \xi_{t}\right)$ denote the maximum attainable value of $U_{t}$ among the state-contingent paths that satisfy the constraints just mentioned, where $X_{t} \equiv\left(F_{t}, K_{t}, W_{t}\right) \cdot{ }^{35}$ Among these constraints is the requirement that

$$
W_{t}=\frac{b_{t-1}}{\prod_{t}} \tilde{u}_{c}\left(Y_{t}-G_{t} ; \xi_{t}\right)
$$

in order for (A.18) to be satisfied. Thus a specified value for $W_{t}$ implies a restriction on the possible values of $\Pi_{t}$ and $Y_{t}$, given the predetermined real debt $b_{t-1}$ and the exogenous disturbances.

The two-stage optimization problem is the following. In the first stage, values of the endogenous variables $x_{t_{0}}$, where $x_{t} \equiv\left(\Pi_{t}, Y_{t}, \tau_{t}, b_{t}, \Delta_{t}\right)$, and state-contingent commitments $X_{t_{0}+1}\left(\xi_{t_{0}+1}\right)$ for the following period, are chosen so as to maximize an objective defined below. In the second stage, the equilibrium evolution from period $t_{0}+1$ onward is chosen to solve the maximization problem that defines the value function $V\left(b_{t_{0}}, \Delta_{t_{0}}, X_{t_{0}+1} ; \xi_{t_{0}+1}\right)$, given the state of the world $\xi_{t_{0}+1}$ and the precommitted values for $X_{t_{0}+1}$ associated with that state.

In defining the objective for the first stage of this equivalent formulation of the Ramsey problem, it is useful to let $\Pi(F, K)$ denote the value of $\Pi_{t}$ that solves (A.16) for given values of $F_{t}$ and $K_{t}$, and to let $s(x ; \xi)$ denote the real primary surplus $s_{t}$ defined by (A.19) in the case of given values of $x_{t}$ and $\xi_{t}$. We also define the functional relationships

$$
\hat{J}[x, X(\cdot)]\left(\xi_{t}\right) \equiv U\left(Y_{t}, \Delta_{t} ; \xi_{t}\right)+\beta E_{t} V\left(b_{t}, \Delta_{t}, X_{t+1} ; \xi_{t+1}\right),
$$

\footnotetext{
${ }^{35}$ As stated in the text, in our notation for the value function $V, \xi_{t}$ denotes not simply the vector of disturbances in period $t$, but all information in period $t$ about current and future disturbances.
} 


$$
\begin{gathered}
\hat{F}[x, X(\cdot)]\left(\xi_{t}\right) \equiv\left(1-\tau_{t}\right) f\left(Y_{t} ; \xi_{t}\right)+\alpha \beta E_{t}\left\{\Pi\left(F_{t+1}, K_{t+1}\right)^{\theta-1} F_{t+1}\right\} \\
\hat{K}[x, X(\cdot)]\left(\xi_{t}\right) \equiv k\left(Y_{t} ; \xi_{t}\right)+\alpha \beta E_{t}\left\{\Pi\left(F_{t+1}, K_{t+1}\right)^{\theta(1+\omega)} K_{t+1}\right\} \\
\hat{W}[x, X(\cdot)]\left(\xi_{t}\right) \equiv \tilde{u}_{c}\left(Y_{t}-G_{t} ; \xi_{t}\right) s\left(x_{t} ; \xi_{t}\right)+\beta E_{t} W_{t+1},
\end{gathered}
$$

where $f(Y ; \xi)$ and $k(Y ; \xi)$ are defined in (A.13) and (A.14).

Then in the first stage, $x_{t_{0}}$ and $X_{t_{0}+1}(\cdot)$ are chosen so as to maximize

$$
\hat{J}\left[x_{t_{0}}, X_{t_{0}+1}(\cdot)\right]\left(\xi_{t_{0}}\right)
$$

over values of $x_{t_{0}}$ and $X_{t_{0}+1}(\cdot)$ such that

(i) $\Pi_{t_{0}}$ and $\Delta_{t_{0}}$ satisfy (A.17);

(ii) the values

$$
\begin{aligned}
& F_{t_{0}}=\hat{F}\left[x_{t_{0}}, X_{t_{0}+1}(\cdot)\right]\left(\xi_{t_{0}}\right), \\
& K_{t_{0}}=\hat{K}\left[x_{t_{0}}, X_{t_{0}+1}(\cdot)\right]\left(\xi_{t_{0}}\right)
\end{aligned}
$$

satisfy

$$
\Pi_{t_{0}}=\Pi\left(F_{t_{0}}, K_{t_{0}}\right)
$$

(iii) the value

$$
W_{t_{0}}=\hat{W}\left[x_{t_{0}}, X_{t_{0}+1}(\cdot)\right]\left(\xi_{t_{0}}\right)
$$

satisfies (A.22) for $t=t_{0}$; and

(iv) the choices $\left(b_{t_{0}}, \Delta_{t_{0}}, X_{t_{0}+1}\right) \in \mathcal{F}$ for each possible state of the world $\xi_{t_{0}+1}$.

These constraints imply that the objective $\hat{J}\left[x_{t_{0}}, X_{t_{0}+1}(\cdot)\right]\left(\xi_{t_{0}}\right)$ is well-defined, and that values $\left(x_{t_{0}}, X_{t_{0}+1}(\cdot)\right)$ are chosen for which the stage two problem will be welldefined, whichever state of the world is realized in period $t_{0}+1$. Furthermore, in the case of any stage-one choices consistent with the above constraints, and any subsequent evolution consistent with the constraints of the stage-two problem, (A.26) implies that (A.16) is satisfied in period $t_{0}$, while (A.22) implies that (A.18) is satisfied in period $t_{0}$. Constraint (i) above implies that (A.17) is also satisfied in period $t_{0}$. Finally, the constraints of the stage-two problem imply that both (A.16), (A.17) and (A.18) are satisfied in each period $t \geq t_{0}+1$; thus the state-contingent evolution that solves the two-stage problem is a rational-expectations equilibrium. Conversely, one can show that any possible rational-expectations equilibrium satisfies all of these constraints.

One can then reformulate the Ramsey problem, replacing the set of requirements for rational-expectations equilibrium by the stage-one constraints plus the stage-two 
constraints. Since no aspect of the evolution from period $t_{0}+1$ onward, other than the specification of $X_{t_{0}+1}(\cdot)$, affects the stage-one constraints, the optimization problem decomposes into the two stages defined above, where the objective (A.23) corresponds to the maximization of $U_{t_{0}}$ in the first stage.

The optimization problem in stage two of this reformulation of the Ramsey problem is of the same form as the Ramsey problem itself, except that there are additional constraints associated with the precommitted values for the elements of $X_{t_{0}+1}\left(\xi_{t_{0}+1}\right)$. Let us consider a problem like the Ramsey problem just defined, looking forward from some period $t_{0}$, except under the constraints that the quantities $X_{t_{0}}$ must take certain given values, where $\left(b_{t_{0}-1}, \Delta_{t_{0}-1}, X_{t_{0}}\right) \in \mathcal{F}$. This constrained problem can similarly be expressed as a two-stage problem of the same form as above, with an identical stage two problem to the one described above. The stage one problem is also identical to stage one of the Ramsey problem, except that now the plan chosen in stage one must be consistent with the given values $X_{t_{0}}$, so that conditions (A.24), (A.25) and (A.27) are now added to the constraints on the possible choices of $\left(x_{t_{0}}, X_{t_{0}+1}(\cdot)\right)$ in stage one. (The stipulation that $\left(b_{t_{0}-1}, \Delta_{t_{0}-1}, X_{t_{0}}\right) \in \mathcal{F}$ implies that the constraint set remains non-empty despite these additional restrictions.)

Stage two of this constrained problem is thus of exactly the same form as the problem itself. Hence the constrained problem has a recursive form. It can be decomposed into an infinite sequence of problems, in which in each period $t,\left(x_{t}, X_{t+1}(\cdot)\right)$ are chosen to maximize $\hat{J}\left[x_{t}, X_{t+1}(\cdot)\right]\left(\xi_{t}\right)$, given the predetermined state variables $\left(b_{t-1}, \Delta_{t-1}\right)$ and the precommitted values $X_{t}$, subject to the constraints that

(i) $\Pi_{t}$ is given by (A.26), $Y_{t}$ is then given by (A.22), and $\Delta_{t}$ is given by (A.17);

(ii) the precommitted values $X_{t}$ are fulfilled, i.e.,

$$
\begin{aligned}
\hat{F}\left[x_{t}, X_{t+1}(\cdot)\right]\left(\xi_{t}\right) & =F_{t}, \\
\hat{K}\left[x_{t}, X_{t+1}(\cdot)\right]\left(\xi_{t}\right) & =K_{t}, \\
\hat{W}\left[x_{t}, X_{t+1}(\cdot)\right]\left(\xi_{t}\right) & =W_{t} ;
\end{aligned}
$$

and

(iii) the choices $\left(b_{t}, \Delta_{t}, X_{t+1}\right) \in \mathcal{F}$ for each possible state of the world $\xi_{t+1}$.

Our aim in the paper is to provide a local characterization of policy that solves this recursive optimization, in the event of small enough disturbances, and initial conditions $\left(b_{t_{0}-1}, \Delta_{t_{0}-1}, X_{t}\right) \in \mathcal{F}$ that are close enough to consistency with the steady state characterized in the next section of this appendix. 


\section{A.3 The deterministic steady state}

Here we show the existence of a steady state, i.e., of an optimal policy (under appropriate initial conditions) of the 'recursive policy problem just defined that involves constant values of all variables. We now consider a deterministic problem in which the exogenous disturbances $\bar{C}_{t}, G_{t}, \bar{H}_{t}, A_{t}, \mu_{t}^{w}, \zeta_{t}$ each take constant values $\bar{C}, \bar{H}, \bar{A}$, $\bar{\mu}^{w}>0$ and $\bar{G}, \bar{\zeta} \geq 0$ for all $t \geq t_{0}$, and we start from initial conditions $b_{t_{0}-1}=\bar{b}>0$. (The value of $\bar{b}$ is arbitrary, subject to an upper bound discussed below.) We wish to find an initial degree of price dispersion $\Delta_{t_{0}-1}$ and initial commitments $X_{t_{0}}=\bar{X}$ such that the recursive (or "stage two") problem involves a constant policy $x_{t_{0}}=\bar{x}$, $X_{t+1}=\bar{X}$ each period, in which $\bar{b}$ is equal to the initial real debt and $\bar{\Delta}$ is equal to the initial price dispersion.

We thus consider the problem of maximizing

$$
U_{t_{0}}=\sum_{t=t_{0}}^{\infty} \beta^{t-t_{0}} U\left(Y_{t}, \Delta_{t}\right)
$$

subject to the constraints

$$
\begin{gathered}
K_{t} p\left(\Pi_{t}\right)^{\frac{1+\omega \theta}{\theta-1}}=F_{t} \\
F_{t}=\left(1-\tau_{t}\right) f\left(Y_{t}\right)+\alpha \beta \Pi_{t+1}^{\theta-1} F_{t+1}, \\
K_{t}=k\left(Y_{t}\right)+\alpha \beta \Pi_{t+1}^{\theta(1+\omega)} K_{t+1}, \\
W_{t}=u_{c}\left(Y_{t}\right)\left(\tau_{t} Y_{t}-\bar{G}-\bar{\zeta}\right)+\beta W_{t+1}, \\
W_{t}=\frac{u_{c}\left(Y_{t}\right) b_{t-1}}{\Pi_{t}}, \\
\Delta_{t}=\alpha \Delta_{t-1} \Pi_{t}^{\theta(1+\omega)}+(1-\alpha) p\left(\Pi_{t}\right)^{-\frac{\theta(1+\omega)}{1-\theta}}
\end{gathered}
$$

and given the specified initial conditions $b_{t_{0}-1}, \Delta_{t_{0}-1}, X_{t_{0}}$, where we have defined

$$
p\left(\Pi_{t}\right) \equiv\left(\frac{1-\alpha \Pi_{t}^{\theta-1}}{1-\alpha}\right)
$$

We introduce Lagrange multipliers $\phi_{1 t}$ through $\phi_{6 t}$ corresponding to constraints (A.32) through (A.37) respectively. We also introduce multipliers dated $t_{0}$ corresponding to the constraints implied by the initial conditions $X_{t_{0}}=\bar{X}$; the latter multipliers are normalized in such a way that the first-order conditions take the same 
form at date $t_{0}$ as at all later dates. The first-order conditions of the maximization problem are then the following. The one with respect to $Y_{t}$ is

$$
\begin{gathered}
U_{y}\left(Y_{t}, \Delta_{t}\right)-\left(1-\tau_{t}\right) f_{y}\left(Y_{t}\right) \phi_{2 t}-k_{y}\left(Y_{t}\right) \phi_{3 t}-\tau_{t} f_{y}\left(Y_{t}\right) \phi_{4 t}+ \\
+u_{c c}\left(Y_{t}\right)(\bar{G}+\bar{\zeta}) \phi_{4 t}-u_{c c}\left(Y_{t}\right) b_{t-1} \Pi_{t}^{-1} \phi_{5 t}=0
\end{gathered}
$$

that with respect to $\Delta_{t}$ is

$$
U_{\Delta}\left(Y_{t}, \Delta_{t}\right)+\phi_{6 t}-\alpha \beta \Pi_{t+1}^{\theta(1+\omega)} \phi_{6, t+1}=0
$$

that with respect to $\Pi_{t}$ is

$$
\begin{gathered}
\frac{1+\omega \theta}{\theta-1} p\left(\Pi_{t}\right)^{\frac{(1+\omega \theta)}{\theta-1}-1} p_{\pi}\left(\Pi_{t}\right) K_{t} \phi_{1, t}-\alpha(\theta-1) \Pi_{t}^{\theta-2} F_{t} \phi_{2, t-1} \\
-\theta(1+\omega) \alpha \Pi_{t}^{\theta(1+\omega)-1} K_{t} \phi_{3, t-1}+u_{c}\left(Y_{t}\right) b_{t-1} \Pi_{t}^{-2} \phi_{5 t}+ \\
-\theta(1+\omega) \alpha \Delta_{t-1} \Pi_{t}^{\theta(1+\omega)-1} \phi_{6 t}-\frac{\theta(1+\omega)}{\theta-1}(1-\alpha) p\left(\Pi_{t}\right)^{\frac{(1+\omega \theta)}{\theta-1}} p_{\pi}\left(\Pi_{t}\right) \phi_{6 t}=0
\end{gathered}
$$

that with respect to $\tau_{t}$ is

$$
\phi_{2 t}-\phi_{4 t}=0
$$

that with respect to $F_{t}$ is

$$
-\phi_{1 t}+\phi_{2 t}-\alpha \Pi_{t}^{\theta-1} \phi_{2, t-1}=0
$$

that with respect to $K_{t}$ is

$$
p\left(\Pi_{t}\right)^{\frac{1+\omega \theta}{\theta-1}} \phi_{1 t}+\phi_{3 t}-\alpha \Pi_{t}^{\theta(1+\omega)} \phi_{3, t-1}=0
$$

that with respect to $W_{t}$ is

$$
\phi_{4 t}-\phi_{4, t-1}+\phi_{5 t}=0
$$

and finally, that with respect to $b_{t}$ is

$$
\phi_{5 t}=0
$$

We search for a solution to these first-order conditions in which $\Pi_{t}=\bar{\Pi}, \Delta_{t}=$ $\bar{\Delta}, Y_{t}=\bar{Y}, \tau_{t}=\bar{\tau}$ and $b_{t}=\bar{b}$ at all times. A steady-state solution of this kind also requires that the Lagrange multipliers take constant values. We furthermore conjecture the existence of a solution in which $\bar{\Pi}=1$, as stated in the text. Note 
that such a solution implies that $\bar{\Delta}=1, p(\bar{\Pi})=1, p_{\pi}(\bar{\Pi})=-(\theta-1) \alpha /(1-\alpha)$, and $\bar{K}=\bar{F}$. Using these substitutions, we find that (the steady-state version of) each of the first-order conditions (A.38) - (A.45) is satisfied if the steady-state values satisfy

$$
\begin{gathered}
\phi_{1}=(1-\alpha) \phi_{2} \\
{\left[f_{y}(\bar{Y})-k_{y}(\bar{Y})-u_{c c}(\bar{Y}-\bar{G})(\bar{G}+\bar{\zeta})\right] \phi_{2}=U_{y}(\bar{Y}, 1)} \\
\phi_{3}=-\phi_{2} \\
\phi_{4}=\phi_{2} \\
\phi_{5}=0 \\
(1-\alpha \beta) \phi_{6}=-U_{\Delta}(\bar{Y}, 1) .
\end{gathered}
$$

These equations can obviously be solved (uniquely) for the steady-state multipliers, given any value $\bar{Y}>0$.

Similarly, (the steady-state versions of) the constraints (A.32) - (A.37) are satisfied if

$$
\begin{gathered}
(1-\bar{\tau}) u_{c}(\bar{Y}-\bar{G})=\frac{\theta}{\theta-1} \bar{\mu}^{w} v_{y}(\bar{Y}), \\
\bar{\tau} \bar{Y}=\bar{G}+\bar{\zeta}+(1-\beta) \bar{b} \\
\bar{K}=\bar{F}=(1-\alpha \beta)^{-1} k(\bar{Y}), \\
\bar{W}=u_{c}(\bar{Y}-\bar{G}) \bar{b}
\end{gathered}
$$

Equations (A.46) - (A.47) provide two equations to solve for the steady-state values $\bar{Y}$ and $\bar{\tau}$. Under standard (Inada-type) boundary conditions on preferences, equation (A.46) has a unique solution $Y_{1}(\tau)>\bar{G}$ for each possible value of $0 \leq \tau<1$; $^{36}$ this value is a decreasing function of $\tau$, and approaches $\bar{G}$ as $\tau$ approaches 1 . We note furthermore that at least in the case of all small enough values of $\bar{G}$, there exists a range of tax rates $0<\tau_{1}<\tau<\tau_{2} \leq 1$ over which $Y_{1}(\tau)>\bar{G} / \tau{ }^{37}$ Given our assumption that $\bar{b}>0$ and that $\bar{G}, \bar{\zeta} \geq 0,(\mathrm{~A} .47)$ is satisfied only by positive values of

\footnotetext{
${ }^{36}$ There is plainly no possibility of positive supply of output by producers in the case that $\tau_{t} \geq 1$ in any period; hence the steady state must involve $\bar{\tau}<1$.

${ }^{37}$ This is true for any tax rate at which $(1-\tau) u_{c}\left(\bar{G}\left(\tau^{-1}-1\right)\right)$ exceeds $(\theta /(\theta-1)) \bar{\mu}^{w} v_{y}(\bar{G} / \tau)$. Fixing any value $0<\tau<1$, our Inada conditions imply that this inequality holds for all small enough values of $\bar{G}$. And if the inequality holds for some $0<\tau<1$, then by continuity it must hold for an open interval of values of $\tau$.
} 
$\bar{\tau}$; and for each $\bar{\tau}>0$, this equation has a unique solution $Y_{2}(\tau)$. We note furthermore that the locus $Y_{1}(\tau)$ is independent of the values of $\bar{\zeta}$ and $\bar{b}$, while $Y_{2}(\tau)$ approaches

$\bar{G} / \tau$ as $\bar{\zeta}$ and $\bar{b}$ approach zero. Fixing the value of $\bar{G}$ (at a value small enough for the interval $\left(\tau_{1}, \tau_{2}\right)$ to exist), we then observe that for any small enough values of $\bar{b}>0$ and $\bar{\zeta} \geq 0$, there exist values $0<\tau<1$ at which $Y_{2}(\tau)<Y_{1}(\tau)$. On the other hand, for all small enough values of $\tau>0, Y_{2}(\tau)>Y_{1}(\tau)$. Thus by continuity, there must exist a value $0<\bar{\tau}<1$ at which $Y_{1}(\bar{\tau})=Y_{2}(\bar{\tau}) .{ }^{38}$ This allows us to obtain a solution for $0<\bar{\tau}<1$ and $\bar{Y}>0$, in the case of any small enough values of $\bar{G}, \bar{\zeta} \geq 0$ and $\bar{b}>0$. The remaining equations can then be solved (uniquely) for $\bar{K}=\bar{F}$ and for $\bar{W}$.

We have thus verified that a constant solution to the first-order conditions exists. With a method to be explained below, we check that this solution is indeed at least a local optimum. Note that as asserted in the text, this deterministic steady state involves zero inflation, and a steady-state tax rate $0<\bar{\tau}<1$.

\section{A.4 A second-order approximation to utility (equations (2.1) and (2.2))}

We derive here equations (2.1) and (2.2) in the main text, taking a second-order approximation to (equation (A.20)) following the treatment in Woodford (2003, chapter $6)$. We start by approximating the expected discounted value of the utility of the representative household

$$
U_{t_{0}}=E_{t_{0}} \sum_{t=t_{0}}^{\infty} \beta^{t-t_{0}}\left[u\left(Y_{t} ; \xi_{t}\right)-\int_{0}^{1} v\left(y_{t}(i) ; \xi_{t}\right) d i\right] .
$$

First we note that

$$
\int_{0}^{1} v\left(y_{t}(i) ; \xi_{t}\right) d i=\frac{\lambda}{1+\nu} \frac{Y_{t}^{1+\omega}}{A_{t}^{1+\omega} \bar{H}_{t}^{\nu}} \Delta_{t}=v\left(Y_{t} ; \xi_{t}\right) \Delta_{t}
$$

where $\Delta_{t}$ is the measure of price dispersion defined in the text. We can then write

\footnotetext{
${ }^{38}$ In fact, there must exist at least two such solutions, since the Inada conditions also imply that $Y_{2}(\tau)>Y_{1}(\tau)$ for all $\tau$ close enough to 1 . These multiple solutions correspond to a "Laffer curve" result, under which two distinct tax rates result in the same equilibrium level of government revenues. We select the lower-tax, higher-output solution as the one around which we compute our Taylor-series expansions; this is clearly the higher-utility solution.
} 
(A.48) as

$$
U_{t_{0}}=E_{t_{0}} \sum_{t=t_{0}}^{\infty} \beta^{t-t_{0}}\left[u\left(Y_{t} ; \xi_{t}\right)-v\left(Y_{t} ; \xi_{t}\right) \Delta_{t}\right] .
$$

The first term in (A.49) can be approximated using a second-order Taylor expansion around the steady state defined in the previous section as

$$
\begin{aligned}
u\left(Y_{t} ; \xi_{t}\right)= & \bar{u}+\bar{u}_{c} \tilde{Y}_{t}+\bar{u}_{\xi} \xi_{t}+\frac{1}{2} \bar{u}_{c c} \tilde{Y}_{t}^{2}+\bar{u}_{c \xi} \xi_{t} \tilde{Y}_{t}+\frac{1}{2} \xi_{t}^{\prime} \bar{u}_{\xi \xi} \xi_{t}+\mathcal{O}\left(\|\xi\|^{3}\right) \\
= & \bar{u}+\bar{Y} \bar{u}_{c} \cdot\left(\hat{Y}_{t}+\frac{1}{2} \hat{Y}_{t}^{2}\right)+\bar{u}_{\xi} \xi_{t}+\frac{1}{2} \bar{Y} \bar{u}_{c c} \hat{Y}_{t}^{2}+ \\
& +\bar{Y} \bar{u}_{c \xi} \xi_{t} \hat{Y}_{t}+\frac{1}{2} \xi_{t}^{\prime} \bar{u}_{\xi \xi} \xi_{t}+\mathcal{O}\left(\|\xi\|^{3}\right) \\
= & \bar{Y} u_{c} \hat{Y}_{t}+\frac{1}{2}\left[\bar{Y} \bar{u}_{c}+\bar{Y}^{2} \bar{u}_{c c} \mid \hat{Y}_{t}^{2}-\bar{Y}^{2} \bar{u}_{c c} g_{t} \hat{Y}_{t}+\text { t.i.p. }+\mathcal{O}\left(\|\xi\|^{3}\right)\right. \\
= & \bar{Y} \bar{u}_{c}\left\{\hat{Y}_{t}+\frac{1}{2}\left(1-\sigma^{-1}\right) \hat{Y}_{t}^{2}+\sigma^{-1} g_{t} \hat{Y}_{t}\right\}+ \\
& + \text { t.i.p. }+\mathcal{O}\left(\|\xi\|^{3}\right),
\end{aligned}
$$

where a bar denotes the steady-state value for each variable, a tilde denotes the deviation of the variable from its steady-state value (e.g., $\tilde{Y}_{t} \equiv Y_{t}-\bar{Y}$ ), and a hat refers to the $\log$ deviation of the variable from its steady-state value (e.g., $\hat{Y}_{t} \equiv \ln Y_{t} / \bar{Y}$ ). We use $\xi_{t}$ to refer to the entire vector of exogenous shocks,

$$
\xi_{t}^{\prime} \equiv\left[\begin{array}{lllll}
\hat{\zeta}_{t} & \hat{G} & g_{t} & q_{t} & \hat{\mu}_{t}^{w}
\end{array}\right],
$$

in which $\hat{\zeta}_{t} \equiv\left(\zeta_{t}-\bar{\zeta}\right) / \bar{Y}, \hat{G}_{t} \equiv\left(G_{t}-\bar{G}\right) / \bar{Y}, g_{t} \equiv \hat{G}_{t}+s_{C} \bar{c}_{t}, \omega q_{t} \equiv \nu \bar{h}_{t}+\phi(1+\nu) a_{t}$, $\hat{\mu}_{t}^{w} \equiv \ln \mu_{t}^{w} / \bar{\mu}^{w}, \bar{c}_{t} \equiv \ln \bar{C}_{t} / \bar{C}, a_{t} \equiv \ln A_{t} / \bar{A}, \bar{h}_{t} \equiv \ln \bar{H}_{t} / \bar{H}$. Moreover, we use the definitions $\sigma^{-1} \equiv \tilde{\sigma}^{-1} s_{C}^{-1}$ with $s_{C} \equiv \bar{C} / \bar{Y}$ and $s_{C}+s_{G}=1$. We have used the Taylor expansion

$$
Y_{t} / \bar{Y}=1+\hat{Y}_{t}+\frac{1}{2} \hat{Y}_{t}^{2}+\mathcal{O}\left(\|\xi\|^{3}\right)
$$

to get a relation for $\tilde{Y}_{t}$ in terms of $\hat{Y}_{t}$. Finally the term "t.i.p." denotes terms that are independent of policy, and may accordingly be suppressed as far as the welfare ranking of alternative policies is concerned. 
We may similarly approximate $v\left(Y_{t} ; \xi_{t}\right) \Delta_{t}$ by

$$
\begin{aligned}
v\left(Y_{t} ; \xi_{t}\right) \Delta_{t}= & \bar{v}+\bar{v}\left(\Delta_{t}-1\right)+\bar{v}_{y}\left(Y_{t}-\bar{Y}\right)+\bar{v}_{y}\left(\Delta_{t}-1\right)\left(Y_{t}-\bar{Y}\right)+\left(\Delta_{t}-1\right) \bar{v}_{\xi} \xi_{t}+ \\
& +\frac{1}{2} \bar{v}_{y y}\left(Y_{t}-\bar{Y}\right)^{2}+\left(Y_{t}-\bar{Y}\right) \bar{v}_{y \xi} \xi_{t}+\mathcal{O}\left(\|\xi\|^{3}\right) \\
= & \bar{v}\left(\Delta_{t}-1\right)+\bar{v}_{y} \bar{Y}\left(\hat{Y}_{t}+\frac{1}{2} \hat{Y}_{t}^{2}\right)+\bar{v}_{y}\left(\Delta_{t}-1\right) \bar{Y}_{t}+\left(\Delta_{t}-1\right) \bar{v}_{\xi} \xi_{t}+ \\
& +\frac{1}{2} \bar{v}_{y y} \bar{Y}^{2} \hat{Y}_{t}^{2}+\bar{Y} \hat{Y}_{t} \bar{v}_{y \xi} \xi_{t}+\text { t.i.p. }+\mathcal{O}\left(\|\xi\|^{3}\right) \\
= & \bar{v}_{y} \bar{Y}\left[\frac{\Delta_{t}-1}{1+\omega}+\hat{Y}_{t}+\frac{1}{2}(1+\omega) \hat{Y}_{t}^{2}+\left(\Delta_{t}-1\right) \hat{Y}_{t}-\omega \hat{Y}_{t} q_{t}+\right. \\
& \left.-\frac{\Delta_{t}-1}{1+\omega} \omega q_{t}\right]+ \text { t.i.p. }+\mathcal{O}\left(\|\xi\|^{3}\right) .
\end{aligned}
$$

We take a second-order expansion of (A.21), obtaining

$$
\hat{\Delta}_{t}=\alpha \hat{\Delta}_{t-1}+\frac{\alpha}{1-\alpha} \theta(1+\omega)(1+\omega \theta) \frac{\pi_{t}^{2}}{2}+\text { t.i.p. }+\mathcal{O}\left(\|\xi\|^{3}\right)
$$

This in turn allows us to approximate $v\left(Y_{t} ; \xi_{t}\right) \Delta_{t}$ as

$$
v\left(Y_{t} ; \xi_{t}\right) \Delta_{t}=(1-\Phi) \bar{Y} u_{c}\left\{\frac{\hat{\Delta}_{t}}{1+\omega}+\hat{Y}_{t}+\frac{1}{2}(1+\omega) \hat{Y}_{t}^{2}-\omega \hat{Y}_{t} q_{t}\right\}+\text { t.i.p. }+\mathcal{O}\left(\|\xi\|^{3}\right),
$$

where we have used the steady state relation $\bar{v}_{y}=(1-\Phi) \bar{u}_{c}$ to replace $\bar{v}_{y}$ by $(1-\Phi) \bar{u}_{c}$, and where

$$
\Phi \equiv 1-\left(\frac{\theta-1}{\theta}\right)\left(\frac{1-\bar{\tau}}{\bar{\mu}^{w}}\right)<1
$$

measures the inefficiency of steady-state output $\bar{Y}$.

Combining (A.50) and (A.52), we finally obtain equation (2.1) in the text,

$$
\begin{aligned}
U_{t_{0}} & =\bar{Y} \bar{u}_{c} \cdot E_{t_{0}} \sum_{t=t_{0}}^{\infty} \beta^{t-t_{0}} \Phi \widehat{Y}_{t}-\frac{1}{2} u_{y y} \widehat{Y}_{t}^{2}+\widehat{Y}_{t} u_{\xi} \xi_{t}-u_{\Delta} \hat{\Delta}_{t} \\
& + \text { t.i.p. }+\mathcal{O}\left(\|\xi\|^{3}\right),
\end{aligned}
$$

where

$$
\begin{aligned}
u_{y y} & \equiv\left(\omega+\sigma^{-1}\right)-(1-\Phi)(1+\omega) \\
u_{\xi} \xi_{t} & \equiv\left[\sigma^{-1} g_{t}+(1-\Phi) \omega q_{t}\right] \\
u_{\Delta} & \equiv \frac{(1-\Phi)}{1+\omega}
\end{aligned}
$$


We finally observe that (A.51) can be integrated to obtain

$$
\sum_{t=t_{0}}^{\infty} \beta^{t} \hat{\Delta}_{t}=\frac{\alpha}{(1-\alpha)(1-\alpha \beta)} \theta(1+\omega)(1+\omega \theta) \sum_{t=t_{0}}^{\infty} \beta^{t} \pi_{t}^{2}+\text { t.i.p. }+\mathcal{O}\left(\|\xi\|^{3}\right) .
$$

By substituting (A.54) into (A.53), we obtain

$$
\begin{aligned}
U_{t_{0}}= & \bar{Y} \bar{u}_{c} \cdot E_{t_{0}} \sum_{t=t_{0}}^{\infty} \beta^{t-t_{0}}\left[\Phi \widehat{Y}_{t}-\frac{1}{2} u_{y y} \widehat{Y}_{t}^{2}+\widehat{Y}_{t} u_{\xi} \xi_{t}-u_{\pi} \pi_{t}^{2}\right] \\
& + \text { t.i.p. }+\mathcal{O}\left(\|\xi\|^{3}\right) .
\end{aligned}
$$

This coincides with equation (2.2) in the text, where we have further defined

$$
\kappa \equiv \frac{(1-\alpha \beta)(1-\alpha)}{\alpha} \frac{\left(\omega+\sigma^{-1}\right)}{(1+\theta \omega)}, \quad u_{\pi} \equiv \frac{\theta\left(\omega+\sigma^{-1}\right)(1-\Phi)}{\kappa} .
$$

\section{A.5 A second-order approximation to the AS equation (equa- tion (1.11))}

We now compute a second-order approximation to the aggregate supply equation (A.16), or equation (1.11) in the main text. We start from (A.10) that can be written as

$$
\tilde{p}_{t}=\left(\frac{K_{t}}{F_{t}}\right)^{\frac{1}{1+\omega \theta}}
$$

where $\widetilde{p}_{t} \equiv p_{t}^{*} / P_{t}$. As shown in Benigno and Woodford (2003), a second-order expansion of this can be expressed in the form

$$
\begin{aligned}
\frac{(1+\omega \theta)}{(1-\alpha \beta)} \hat{\tilde{p}}_{t}= & z_{t}+\alpha \beta \frac{(1+\omega \theta)}{(1-\alpha \beta)} E_{t}\left(\hat{\tilde{p}}_{t+1}-\hat{P}_{t, t+1}\right)+\frac{1}{2} z_{t} X_{t}+ \\
& -\frac{1}{2}(1+\omega \theta) \hat{\tilde{p}}_{t} Z_{t}+\frac{1}{2} \alpha \beta(1+\omega \theta) E_{t}\left\{\left(\hat{\tilde{p}}_{t+1}-\hat{P}_{t, t+1}\right) Z_{t+1}\right\} \\
& +\frac{\alpha \beta}{2(1-\alpha \beta)}(1-2 \theta-\omega \theta)(1+\omega \theta) E_{t}\left\{\left(\hat{\tilde{p}}_{t+1}-\hat{P}_{t, t+1}\right) \hat{P}_{t, t+1}\right\} \\
& \quad+\text { s.o.t.i.p. }+\mathcal{O}\left(\|\xi\|^{3}\right),
\end{aligned}
$$

where we define

$$
\begin{gathered}
\hat{P}_{t, T} \equiv \log \left(P_{t} / P_{T}\right), \\
z_{t} \equiv \omega\left(\hat{Y}_{t}-q_{t}\right)+\tilde{\sigma}^{-1}\left(\hat{C}_{t}-\bar{c}_{t}\right)-\hat{S}_{t}+\hat{\mu}_{t}^{w},
\end{gathered}
$$




$$
Z_{t} \equiv E_{t}\left\{\sum_{T=t}^{+\infty}(\alpha \beta)^{T-t}\left[X_{T}+(1-2 \theta-\omega \theta) \hat{P}_{t, T}\right]\right\}
$$

and in this last expression

$$
X_{T} \equiv(2+\omega) \hat{Y}_{T}-\omega q_{T}+\hat{\mu}_{T}^{w}+\hat{S}_{T}-\tilde{\sigma}^{-1}\left(\hat{C}_{T}-\bar{c}_{T}\right)
$$

where $\hat{S}_{t}=\ln \left(1-\tau_{t}\right) /(1-\bar{\tau})$. Here "s.o.t.i.p." refers to second-order (or higher) terms independent of policy; the first-order terms have been kept as these will matter for the log-linear aggregate-supply relation that appears as a constraint in our policy problem.

We next take a second-order expansion of the law of motion (A.15) for the price index, obtaining

$$
\hat{\tilde{p}}_{t}=\frac{\alpha}{1-\alpha} \pi_{t}-\frac{1-\theta}{2} \frac{\alpha}{(1-\alpha)^{2}} \pi_{t}^{2}+\mathcal{O}\left(\|\xi\|^{3}\right)
$$

where we have used the fact that

$$
\hat{\tilde{p}}_{t}=\frac{\alpha}{1-\alpha} \pi_{t}+\mathcal{O}\left(\|\xi\|^{2}\right)
$$

and $\hat{P}_{t-1, t}=-\pi_{t}$. We can then plug (A.56) into (A.55) obtaining

$$
\begin{aligned}
\pi_{t}= & \frac{1-\theta}{2} \frac{1}{(1-\alpha)} \pi_{t}^{2}+\frac{\kappa}{\left(\omega+\sigma^{-1}\right)} z_{t}+\beta E_{t} \pi_{t+1}-\frac{1-\theta}{2} \frac{\alpha \beta}{(1-\alpha)} E_{t} \pi_{t+1}^{2} \\
& +\frac{1}{2} \frac{\kappa}{\left(\omega+\sigma^{-1}\right)} z_{t} X_{t}-\frac{1}{2}(1-\alpha \beta) \pi_{t} Z_{t}+\frac{\beta}{2}(1-\alpha \beta) E_{t}\left\{\pi_{t+1} Z_{t+1}\right\} \\
& -\frac{\beta}{2}(1-2 \theta-\omega \theta) E_{t}\left\{\pi_{t+1}^{2}\right\}+\text { s.o.t.i.p. }+\mathcal{O}\left(\|\xi\|^{3}\right) .
\end{aligned}
$$

We note further that a second-order approximation to the identity $C_{t}=Y_{t}-G_{t}$ yields

$$
\hat{C}_{t}=s_{C}^{-1} \hat{Y}_{t}-s_{C}^{-1} \hat{G}_{t}+\frac{s_{C}^{-1}\left(1-s_{C}^{-1}\right)}{2} \hat{Y}_{t}^{2}+s_{C}^{-2} \hat{Y}_{t} \hat{G}_{t}+\text { s.o.t.i.p. }+\mathcal{O}\left(\|\xi\|^{3}\right),
$$

and that

$$
\hat{S}_{t}=-\omega_{\tau} \hat{\tau}_{t}-\frac{\omega_{\tau}}{(1-\bar{\tau})} \hat{\tau}_{t}^{2}+\mathcal{O}\left(\|\xi\|^{3}\right)
$$

where $\omega_{\tau} \equiv \bar{\tau} /(1-\bar{\tau})$. By substituting (A.58) and (A.59) into the definition of $z_{t}$ in (A.57), we finally obtain a quadratic approximation to the AS relation. 
This can be expressed compactly in the form

$$
\begin{aligned}
V_{t}= & \kappa\left(c_{x}^{\prime} x_{t}+c_{\xi}^{\prime} \xi_{t}+\frac{1}{2} x_{t}^{\prime} C_{x} x_{t}+x_{t}^{\prime} C_{\xi} \xi_{t}+\frac{1}{2} c_{\pi} \pi_{t}^{2}\right)+\beta E_{t} V_{t+1} \\
& + \text { s.o.t.i.p. }+\mathcal{O}\left(\|\xi\|^{3}\right)
\end{aligned}
$$

where we have defined

$$
\begin{aligned}
& x_{t} \equiv\left[\begin{array}{c}
\hat{\tau}_{t} \\
\hat{Y}_{t}
\end{array}\right], \\
& c_{x}^{\prime}=\left[\begin{array}{ll}
\psi & 1
\end{array}\right] \text {, }
\end{aligned}
$$

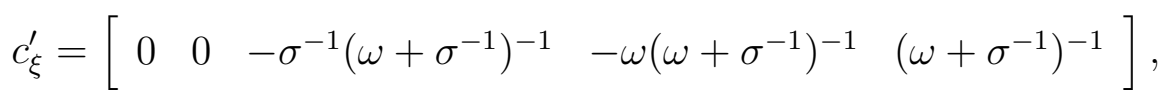

$$
\begin{aligned}
& C_{x}=\left[\begin{array}{cc}
\psi & \left(1-\sigma^{-1}\right) \psi \\
\left(1-\sigma^{-1}\right) \psi & \left(2+\omega-\sigma^{-1}\right)+\sigma^{-1}\left(1-s_{C}^{-1}\right)\left(\omega+\sigma^{-1}\right)^{-1}
\end{array}\right], \\
& C_{\xi}=\left[\begin{array}{ccccc}
0 & 0 & \psi \sigma^{-1} & 0 & 0 \\
0 & \frac{\sigma^{-1} s_{C}^{-1}}{\left(\omega+\sigma^{-1}\right)} & -\frac{\sigma^{-1}\left(1-\sigma^{-1}\right)}{\left(\omega+\sigma^{-1}\right)} & -\frac{\omega(1+\omega)}{\left(\omega+\sigma^{-1}\right)} & \frac{(1+\omega)}{\left(\omega+\sigma^{-1}\right)}
\end{array}\right], \\
& c_{\pi}=\frac{\theta(1+\omega)\left(\omega+\sigma^{-1}\right)}{\kappa} \\
& V_{t}=\pi_{t}+\frac{1}{2} v_{\pi} \pi_{t}^{2}+v_{z} \pi_{t} Z_{t}, \\
& Z_{t}=z_{x}^{\prime} x_{t}+z_{\pi} \pi_{t}+z_{\xi}^{\prime} \xi_{t}+\alpha \beta E_{t} Z_{t+1}
\end{aligned}
$$

in which the coefficients

$$
\psi \equiv \omega_{\tau} /\left(\omega+\sigma^{-1}\right),
$$

and

$$
\begin{aligned}
& v_{\pi} \equiv \theta(1+\omega)-\frac{1-\theta}{(1-\alpha)}, \quad v_{z} \equiv \frac{(1-\alpha \beta)}{2}, \\
& v_{k} \equiv \frac{\alpha \beta}{1-\alpha \beta}(1-2 \theta-\omega \theta), \\
& z_{x}^{\prime} \equiv\left[\begin{array}{ll}
\left(2+\omega-\sigma^{-1}\right)+v_{k}\left(\omega+\sigma^{-1}\right) & -\omega_{\tau}\left(1-v_{k}\right)
\end{array}\right] \text {, } \\
& z_{\xi}^{\prime} \equiv\left[\begin{array}{lllll}
0 & 0 & \sigma^{-1}\left(1-v_{k}\right) & -\omega\left(1+v_{k}\right) & \left(1-v_{k}\right)
\end{array}\right], \\
& z_{\pi} \equiv-v_{k} .
\end{aligned}
$$


Note that in a first-order approximation, (A.60) can be written as simply

$$
\pi_{t}=\kappa\left[\hat{Y}_{t}+\psi \hat{\tau}_{t}+c_{\xi}^{\prime} \xi_{t}\right]+\beta E_{t} \pi_{t+1}
$$

where

$$
c_{\xi}^{\prime} \xi_{t} \equiv\left(\omega+\sigma^{-1}\right)^{-1}\left[-\sigma^{-1} g_{t}-\omega q_{t}+\hat{\mu}_{t}^{w}\right] .
$$

We can also integrate (A.60) forward from time $t_{0}$ to obtain

$$
\begin{aligned}
V_{t_{0}}= & E_{t_{0}} \sum_{t=t_{0}}^{\infty} \beta^{t-t_{0}} \kappa\left(c_{x}^{\prime} x_{t}+\frac{1}{2} x_{t}^{\prime} C_{x} x_{t}+x_{t}^{\prime} C_{\xi} \xi_{t}+\frac{1}{2} c_{\pi} \pi_{t}^{2}\right) \\
& + \text { t.i.p. }+\mathcal{O}\left(\|\xi\|^{3}\right),
\end{aligned}
$$

where the term $c_{\xi}^{\prime} \xi_{t}$ is now included in terms independent of policy. (Such terms matter when part of the log-linear constraints, as in the case of (A.61), but not when part of the quadratic objective.)

\section{A.6 A second-order approximation to the intertemporal gov- ernment solvency condition (equation (1.15))}

We now derive a second-order approximation to the intertemporal government solvency condition. We use the definition

$$
W_{t} \equiv E_{t} \sum_{T=t}^{\infty} \beta^{T-t} \tilde{u}_{c}\left(Y_{T} ; \xi_{T}\right) s_{T}
$$

where

$$
s_{t} \equiv \tau_{t} Y_{t}-G_{t}-\zeta_{t}
$$

and

$$
W_{t}=\frac{b_{t-1}}{\Pi_{t}} \tilde{u}_{c}\left(C_{t} ; \xi_{t}\right)
$$


First, we take a second-order approximation of the term $\tilde{u}_{c}\left(C_{t} ; \xi_{t}\right) s_{t}$ obtaining

$$
\begin{aligned}
\tilde{u}_{c}\left(C_{t} ; \xi_{t}\right) s_{t}= & \bar{s} \overline{\tilde{u}}_{c}+\overline{\tilde{u}}_{c c} \bar{s} \tilde{C}_{t}+\overline{\tilde{u}}_{c} \tilde{s}_{t}+\bar{s} \overline{\tilde{u}}_{c \xi} \xi_{t}+\frac{1}{2} \bar{s} \overline{\tilde{u}}_{c c c} \tilde{C}_{t}^{2}+\overline{\tilde{u}}_{c c} \tilde{C}_{t} \tilde{s}_{t}+\bar{s} \tilde{C}_{t} \overline{\tilde{u}}_{c c \xi} \xi_{t} \\
& +\tilde{s}_{t} \overline{\tilde{u}}_{c \xi} \xi_{t}+\text { s.o.t.i.p. }+\mathcal{O}\left(\|\xi\|^{3}\right), \\
= & \bar{s} \overline{\tilde{u}}_{c}+\overline{\tilde{u}}_{c c} \bar{s} \bar{C} \hat{C}_{t}+\overline{\tilde{u}}_{c} \tilde{s}_{t}+\bar{s} \overline{\tilde{u}}_{c \xi} \xi_{t}+\frac{1}{2} \bar{s}\left(\overline{\tilde{u}}_{c c} \bar{C}+\overline{\tilde{u}}_{c c c} \bar{C}^{2}\right) \hat{C}_{t}^{2}+\bar{C} \overline{\tilde{u}}_{c c} \hat{C}_{t} \tilde{s}_{t}+ \\
& \bar{s} \bar{C}_{\tilde{u}_{c c \xi}} \xi_{t} \hat{C}_{t}+\overline{\tilde{u}}_{c \xi} \xi_{t} \tilde{s}_{t}+\text { s.o.t.i.p. }+\mathcal{O}\left(\|\xi\|^{3}\right) \\
= & \bar{s} \overline{\tilde{u}}_{c}+\overline{\tilde{u}}_{c}\left[-\widetilde{\sigma}^{-1} \bar{s} \hat{C}_{t}+\tilde{s}_{t}+\bar{s} \overline{\tilde{u}}_{c}^{-1} \overline{\tilde{u}}_{c \xi} \xi_{t}+\frac{1}{2} \bar{s} \tilde{\sigma}^{-2} \tilde{C}_{t}^{2}-\tilde{\sigma}^{-1} \tilde{s}_{t} \hat{C}_{t}+\right. \\
& \left.\bar{s} \bar{C}_{c}^{-1} \overline{\tilde{u}}_{c c \xi} \xi_{t} \hat{C}_{t}+\overline{\tilde{u}}_{c}^{-1} \overline{\tilde{u}}_{c \xi} \xi_{t} \tilde{s}_{t}\right]+ \text { s.o.t.i.p. }+\mathcal{O}\left(\|\xi\|^{3}\right) \\
= & \bar{s} \overline{\tilde{u}}_{c}+\overline{\tilde{u}}_{c}\left[-\tilde{\sigma}^{-1} \bar{s}\left(\hat{C}_{t}-\bar{c}_{t}\right)+\tilde{s}_{t}+\frac{1}{2} \bar{s} \tilde{\sigma}^{-2} \tilde{C}_{t}^{2}-\tilde{\sigma}^{-1} \tilde{s}_{t}\left(\hat{C}_{t}-\bar{c}_{t}\right)-\tilde{\sigma}^{-2} \bar{s}_{t} \hat{C}_{t}\right] \\
& + \text { s.o.t.i.p. }+\mathcal{O}\left(\|\xi\|^{3}\right)
\end{aligned}
$$

where we have followed previous definitions and use the isoelastic functional forms assumed and note that we can write $\overline{\tilde{u}}_{c}^{-1} \overline{\tilde{u}}_{c \xi} \xi_{t}=\tilde{\sigma}^{-1} \bar{c}_{t}$ and $\bar{C} \overline{\tilde{u}}_{c}^{-1} \overline{\tilde{u}}_{c c \xi} \xi_{t}=-\tilde{\sigma}^{-2} \bar{c}_{t}$. Plugging (A.58) into (A.66) we obtain

$$
\begin{aligned}
\tilde{u}_{c}\left(C_{t} ; \tilde{\xi}_{t}\right) s_{t}= & \bar{s} \overline{\tilde{u}}_{c}\left[1-\sigma^{-1} \hat{Y}_{t}+\sigma^{-1} g_{t}+\bar{s}^{-1} \tilde{s}_{t}+\frac{1}{2}\left[\sigma^{-1}\left(s_{C}^{-1}-1\right)+\sigma^{-2}\right] \hat{Y}_{t}^{2}+\right. \\
& \left.-\sigma^{-1} \bar{s}^{-1}\left(\hat{Y}_{t}-g_{t}\right) \tilde{s}_{t}-\sigma^{-1}\left(s_{C}^{-1} \hat{G}_{t}+\sigma^{-1} g_{t}\right) \hat{Y}_{t}\right]+ \text { s.o.t.i.p. }+ \\
& +\mathcal{O}\left(\|\xi\|^{3}\right)
\end{aligned}
$$

by using previous definitions.

We recall now that the primary surplus is defined as

$$
s_{t}=\tau_{t} Y_{t}-G_{t}-\zeta_{t}
$$

which can be expanded in a second-order expansion to get

$$
\begin{aligned}
\bar{s}^{-1} \tilde{s}_{t}= & \left(1+\omega_{g}\right)\left(\hat{Y}_{t}+\hat{\tau}_{t}\right)-s_{d}^{-1}\left(\hat{G}_{t}+\hat{\zeta}_{t}\right)+\frac{\left(1+\omega_{g}\right)}{2}\left(\hat{Y}_{t}+\hat{\tau}_{t}\right)^{2} \\
& + \text { s.o.t.i.p. }+\mathcal{O}\left(\|\xi\|^{3}\right),
\end{aligned}
$$

where we have defined $s_{d} \equiv \bar{s} / \bar{Y}, \omega_{g}=(\bar{G}+\bar{\zeta}) / \bar{s}$ and $\hat{\zeta}_{t}=\left(\zeta_{t}-\bar{\zeta}\right) / \bar{Y}$. Substituting 
(A.68) into (A.67), we obtain

$$
\begin{aligned}
\tilde{u}_{c}\left(C_{t} ; \tilde{\xi}_{t}\right) s_{t}= & \bar{s} \overline{\tilde{u}}_{c}\left[1-\sigma^{-1} \hat{Y}_{t}+\left(1+\omega_{g}\right)\left(\hat{Y}_{t}+\hat{\tau}_{t}\right)+\sigma^{-1} g_{t}-s_{d}^{-1}\left(\hat{G}_{t}+\hat{\zeta}_{t}\right)\right. \\
& +\frac{\left(1+\omega_{g}\right)}{2} \hat{\tau}_{t}^{2}+\left(1+\omega_{g}\right)\left(1-\sigma^{-1}\right) \hat{\tau}_{t} \hat{Y}_{t}+ \\
& +\frac{1}{2}\left[1+\omega_{g}+\sigma^{-1}\left(s_{C}^{-1}-1\right)+\sigma^{-2}-2 \sigma^{-1}\left(1+\omega_{g}\right)\right] \hat{Y}_{t}^{2}+ \\
& -\sigma^{-1}\left[s_{C}^{-1} \hat{G}_{t}+\left(\sigma^{-1}-1-\omega_{g}\right) g_{t}-s_{d}^{-1}\left(\hat{G}_{t}+\hat{\zeta}_{t}\right)\right] \widehat{Y}_{t} \\
& \left.+\sigma^{-1}\left(1+\omega_{g}\right) g_{t} \hat{\tau}_{t}\right]+ \text { s.o.t.i.p. }+\mathcal{O}\left(\|\xi\|^{3}\right) .
\end{aligned}
$$

Substituting (A.69) into (A.63), we obtain

$$
\begin{aligned}
\tilde{W}_{t}= & (1-\beta)\left[b_{x}^{\prime} x_{t}+b_{\xi}^{\prime} \xi_{t}+\frac{1}{2} x_{t}^{\prime} B_{x} x_{t}+x_{t}^{\prime} B_{\xi} \xi_{t}\right]+\beta E_{t} \tilde{W}_{t+1} \\
& \text { s.o.t.i.p. }+\mathcal{O}\left(\|\xi\|^{3}\right)
\end{aligned}
$$

where $\tilde{W}_{t} \equiv\left(W_{t}-\bar{W}\right) / \bar{W}$ and

$$
\begin{aligned}
& b_{x}^{\prime}=\left[\begin{array}{ll}
\left(1+\omega_{g}\right) & \left(1+\omega_{g}\right)-\sigma^{-1}
\end{array}\right], \\
& b_{\xi}^{\prime}=\left[\begin{array}{lllll}
-s_{d}^{-1} & -s_{d}^{-1} & \sigma^{-1} & 0 & 0
\end{array}\right], \\
& B_{x}=\left[\begin{array}{cc}
\left(1+\omega_{g}\right) & \left(1-\sigma^{-1}\right)\left(1+\omega_{g}\right) \\
\left(1-\sigma^{-1}\right)\left(1+\omega_{g}\right) & \left(1+\omega_{g}\right)+\left(s_{C}^{-1}-1\right) \sigma^{-1}+\sigma^{-2}-2 \sigma^{-1}\left(1+\omega_{g}\right)
\end{array}\right], \\
& B_{\xi}=\left[\begin{array}{ccccc}
0 & 0 & \sigma^{-1}\left(1+\omega_{g}\right) & 0 & 0 \\
s_{d}^{-1} \sigma^{-1} & s_{d}^{-1} \sigma^{-1}-s_{C}^{-1} \sigma^{-1} & -\sigma^{-1}\left(\sigma^{-1}-1-\omega_{g}\right) & 0 & 0
\end{array}\right],
\end{aligned}
$$

We further note from (A.70) that

$$
\tilde{W}_{t} \equiv\left(\hat{b}_{t-1}-\pi_{t}-\widetilde{\sigma}^{-1} \hat{C}_{t}+\bar{c}_{t}\right)+\frac{1}{2}\left(\hat{b}_{t-1}-\pi_{t}-\widetilde{\sigma}^{-1} \hat{C}_{t}+\bar{c}_{t}\right)^{2}+\mathcal{O}\left(\|\xi\|^{3}\right) .
$$

Substituting in (A.58), we obtain

$$
\begin{aligned}
\tilde{W}_{t} \equiv & \hat{b}_{t-1}-\pi_{t}-\sigma^{-1}\left(\hat{Y}_{t}-g_{t}\right)-\frac{\sigma^{-1}\left(1-s_{C}^{-1}\right)}{2} \hat{Y}_{t}^{2}-\sigma^{-1} s_{C}^{-1} \hat{Y}_{t} \hat{G}_{t} \\
& +\frac{1}{2}\left(\hat{b}_{t-1}-\pi_{t}-\sigma^{-1}\left(\hat{Y}_{t}-g_{t}\right)\right)^{2}+\text { s.o.t.i.p. }+\mathcal{O}\left(\|\xi\|^{3}\right)
\end{aligned}
$$

which can be written as

$$
\begin{aligned}
\tilde{W}_{t}= & \hat{b}_{t-1}-\pi_{t}+w_{x}^{\prime} x_{t}+w_{\xi}^{\prime} \xi_{t}+\frac{1}{2} x_{t}^{\prime} W_{x} x_{t}+x_{t}^{\prime} W_{\xi} \xi_{t}+\frac{1}{2}\left[\hat{b}_{t-1}-\pi_{t}+w_{x}^{\prime} x_{t}+w_{\xi}^{\prime} \xi_{t}\right]^{2} \\
& + \text { s.o.t.i.p. }+\mathcal{O}\left(\|\xi\|^{3}\right)
\end{aligned}
$$




$$
\begin{gathered}
w_{x}^{\prime}=\left[\begin{array}{cc}
0 & -\sigma^{-1}
\end{array}\right], \\
w_{\xi}^{\prime}=\left[\begin{array}{ccccc}
0 & 0 & \sigma^{-1} & 0 & 0
\end{array}\right], \\
W_{x}=\left[\begin{array}{cccc}
0 & 0 & \\
0 & \left(s_{C}^{-1}-1\right) \sigma^{-1}
\end{array}\right], \\
W_{\xi}=\left[\begin{array}{ccccc}
0 & 0 & 0 & 0 & 0 \\
0 & -s_{C}^{-1} \sigma^{-1} & 0 & 0 & 0
\end{array}\right] .
\end{gathered}
$$

Note that in the first-order approximation we can simply write (A.70) as

$$
\begin{aligned}
\hat{b}_{t-1}-\pi_{t} & +w_{x}^{\prime} x_{t}+w_{\xi}^{\prime} \xi_{t}=(1-\beta)\left[b_{x}^{\prime} x_{t}+b_{\xi}^{\prime} \xi_{t}\right] \\
& +\beta E_{t}\left[\hat{b}_{t}-\pi_{t+1}+w_{x}^{\prime} x_{t+1}+w_{\xi}^{\prime} \xi_{t+1}\right] .
\end{aligned}
$$

Moreover integrating forward (A.70), we obtain that

$$
\tilde{W}_{t_{0}}=(1-\beta) E_{t_{0}} \sum_{t=t_{0}}^{\infty} \beta^{t-t_{0}}\left[b_{x}^{\prime} x_{t}+\frac{1}{2} x_{t}^{\prime} B_{x} x_{t}+x_{t}^{\prime} B_{\xi} \xi_{t}\right]+\text { t.i.p. }+\mathcal{O}\left(\|\xi\|^{3}\right),
$$

where we have moved $b_{\xi}^{\prime} \xi_{t}$ in t.i.p.

\section{A.7 A quadratic policy objective (equations (2.3) and (2.4))}

We now derive a quadratic approximation to the policy objective function. To this end, we combine equation (A.62) and (A.72) in a way to eliminate the linear term in (A.53). Indeed, we find $\vartheta_{1}, \vartheta_{2}$ such that

$$
\vartheta_{1} b_{x}^{\prime}+\vartheta_{2} c_{x}^{\prime}=a_{x}^{\prime} \equiv[0 \Phi] .
$$

The solution is given by

$$
\begin{aligned}
& \vartheta_{1}=-\frac{\Phi \omega_{\tau}}{\Gamma}, \\
& \vartheta_{2}=\frac{\Phi\left(1+\omega_{g}\right)}{\Gamma},
\end{aligned}
$$

where

$$
\Gamma=\left(\omega+\sigma^{-1}\right)\left(1+\omega_{g}\right)-\omega_{\tau}\left(1+\omega_{g}\right)+\omega_{\tau} \sigma^{-1} .
$$


We can write

$$
\begin{aligned}
E_{t_{0}} \sum_{t=t_{0}}^{\infty} \beta^{t-t_{0}} \Phi \hat{Y}_{t}= & E_{t_{0}} \sum_{t=t_{0}}^{\infty} \beta^{t-t_{0}}\left[\vartheta_{1} b_{x}^{\prime}+\vartheta_{2} c_{x}^{\prime}\right] x_{t} \\
= & -E_{t_{0}} \sum_{t=t_{0}}^{\infty} \beta^{t-t_{0}}\left[\frac{1}{2} x_{t}^{\prime} D_{x} x_{t}+x_{t}^{\prime} D_{\xi} \xi_{t}+\frac{1}{2} d_{\pi} \pi_{t}^{2}\right] \\
& +\vartheta_{1} \tilde{W}_{t_{0}}+\vartheta_{2} \kappa^{-1} V_{t_{0}}+\text { t.i.p. }+\mathcal{O}\left(\|\xi\|^{3}\right)
\end{aligned}
$$

where

$$
D_{x} \equiv \vartheta_{1} B_{x}+\vartheta_{2} C_{x}, \quad \text { etc. }
$$

Hence

$$
\begin{aligned}
U_{t_{0}}= & \Omega E_{t_{0}} \sum_{t=t_{0}}^{\infty} \beta^{t-t_{0}}\left\{a_{x}^{\prime} x_{t}-\frac{1}{2} x_{t}^{\prime} A_{x} x_{t}-x_{t}^{\prime} A_{\xi} \xi_{t}-\frac{1}{2} a_{\pi} \pi_{t}^{2}\right\}+\text { t.i.p. }+\mathcal{O}\left(\|\xi\|^{3}\right) \\
= & -\Omega E_{t_{0}} \sum_{t=t_{0}}^{\infty} \beta^{t-t_{0}}\left\{\frac{1}{2} x_{t}^{\prime} Q_{x} x_{t}+x_{t}^{\prime} Q_{\xi} \xi_{t}+\frac{1}{2} q_{\pi} \pi_{t}^{2}\right\}+T_{t_{0}}+\text { t.i.p. }+\mathcal{O}\left(\|\xi\|^{3}\right) \\
= & -\Omega E_{t_{0}} \sum_{t=t_{0}}^{\infty} \beta^{t-t_{0}}\left\{\frac{1}{2} q_{y}\left(\hat{Y}_{t}-\hat{Y}_{t}^{*}\right)^{2}+\frac{1}{2} q_{\pi} \pi_{t}^{2}\right\}+T_{t_{0}}+ \\
& + \text { t.i.p. }+\mathcal{O}\left(\|\xi\|^{3}\right)
\end{aligned}
$$

In particular, we obtain that $\Omega=\bar{u}_{c} \bar{Y}$ and that

$$
Q_{x}=\left[\begin{array}{cc}
0 & 0 \\
0 & q_{y}
\end{array}\right]
$$

with

$$
\begin{aligned}
q_{y} \equiv & (1-\Phi)\left(\omega+\sigma^{-1}\right)+\Phi\left(\omega+\sigma^{-1}\right) \frac{\left(1+\omega_{g}\right)(1+\omega)}{\Gamma}+\Phi \sigma^{-1} \frac{\left(1+\omega_{\tau}\right)\left(1+\omega_{g}\right)}{\Gamma} \\
& -\Phi \sigma^{-1} s_{C}^{-1} \frac{1+\omega_{g}+\omega_{\tau}}{\Gamma} ;
\end{aligned}
$$

moreover we have defined

$$
Q_{\xi}=\left[\begin{array}{ccccc}
0 & 0 & 0 & 0 & 0 \\
q_{\xi 1} & q_{\xi 2} & q_{\xi 3} & q_{\xi 4} & q_{\xi 5}
\end{array}\right],
$$


with

$$
\begin{gathered}
q_{\xi 1}=-\frac{\Phi \omega_{\tau}}{\Gamma} s_{d}^{-1} \sigma^{-1}, \\
q_{\xi 2}=-\frac{\Phi \sigma^{-1} s_{d}^{-1} \omega_{\tau}}{\Gamma}+\frac{\sigma^{-1} s_{C}^{-1} \Phi\left(1+\omega_{g}+\omega_{\tau}\right)}{\Gamma}, \\
q_{\xi 3}=-(1-\Phi) \sigma^{-1}-\frac{\sigma^{-1} \Phi(1+\omega)\left(1+\omega_{g}\right)}{\Gamma}, \\
q_{\xi 4}=-(1-\Phi) \omega-\frac{\omega \Phi(1+\omega)\left(1+\omega_{g}\right)}{\Gamma}, \\
q_{\xi 5}=\Phi \frac{1+\omega_{g}}{\Gamma}(1+\omega),
\end{gathered}
$$

and

$$
q_{\pi}=\frac{\Phi\left(1+\omega_{g}\right) \theta(1+\omega)\left(\omega+\sigma^{-1}\right)}{\Gamma \kappa}+\frac{(1-\Phi) \theta\left(\omega+\sigma^{-1}\right)}{\kappa} .
$$

We have further defined $\hat{Y}_{t}^{*}$, the desired level of output, as

$$
\hat{Y}_{t}^{*}=-q_{y}^{-1} q_{\xi}^{\prime} \xi_{t}
$$

Finally,

$$
T_{t_{0}} \equiv \bar{Y} \bar{u}_{c}\left[\vartheta_{1} \tilde{W}_{t_{0}}+\vartheta_{2} \kappa^{-1} V_{t_{0}}\right]
$$

is a transitory component. Equation (A.73) corresponds to equation (2.3) in the main text. In particular, given the commitments on the initial values of the vector $X_{t_{0}}$, $W_{t_{0}}$ implies that $\tilde{W}_{t_{0}}$ is given when characterizing the optimal policy from a timeless perspective. Moreover, $F_{t_{0}}$ and $K_{t_{0}}$ imply that $V_{t_{0}}$ and $Z_{t_{0}}$ are as well given. It follows that the value of the transitory component $T_{t_{0}}$ is predetermined under the stage two of the Ramsey problem. Hence, over the set of admissible policies, higher values of (A.73) correspond to lower values of

$$
E_{t_{0}} \sum_{t=t_{0}}^{\infty} \beta^{t-t_{0}}\left\{\frac{1}{2} q_{y}\left(\hat{Y}_{t}-\hat{Y}_{t}^{*}\right)^{2}+\frac{1}{2} q_{\pi} \pi_{t}^{2}\right\} .
$$

It follows that we may rank policies in terms of the implied value of the discounted quadratic loss function (A.74) which corresponds to equation (2.4) in the main text. Because this loss function is purely quadratic (i.e., lacking linear terms), it is possible to evaluate it to second order using only a first-order approximation to the equilibrium evolution of inflation and output under a given policy. Hence the log-linear approximate structural relations (A.61) and (A.71) are sufficiently accurate for our 
purposes. Similarly, it suffices that we use log-linear approximations to the variables $V_{t_{0}}$ and $\tilde{W}_{t_{0}}$ in describing the initial commitments, which are given by

$$
\begin{aligned}
\hat{V}_{t_{0}} & =\pi_{t_{0}} \\
\hat{W}_{t_{0}} & =\hat{b}_{t_{0}-1}-\pi_{t_{0}}+w_{x}^{\prime} x_{t_{0}}+w_{\xi}^{\prime} \xi_{t_{0}} \\
& =\hat{b}_{t_{0}-1}-\pi_{t_{0}}-\sigma^{-1}\left(\hat{Y}_{t_{0}}-g_{t_{0}}\right) .
\end{aligned}
$$

Then an optimal policy from a timeless perspective is a policy from date $t_{0}$ onward that minimizes the quadratic loss function (A.74) subject to the constraints implied by the linear structural relations (A.61) and (A.71) holding in each period $t \geq t_{0}$, given the initial values $\hat{b}_{t_{0}-1}, \hat{\Delta}_{t_{0}-1}$, and subject also to the constraints that certain predetermined values for $\hat{V}_{t_{0}}$ and $\hat{W}_{t_{0}}$ (or alternatively, for $\pi_{t_{0}}$ and for $\hat{Y}_{t_{0}}$ ) be achieved. ${ }^{39}$ We note that under the assumption that $\omega+\sigma^{-1}>\omega_{\tau}=\bar{\tau} /(1-\bar{\tau})$, $\Gamma>0$, which implies that $q_{\pi}>0$. Moreover, if

$$
s_{C}>\frac{\Phi \sigma^{-1}\left(1+\omega_{g}+\omega_{\tau}\right)}{(1-\Phi)\left(\omega+\sigma^{-1}\right) \Gamma+\Phi\left(\omega+\sigma^{-1}\right)\left(1+\omega_{g}\right)(1+\omega)+\Phi \sigma^{-1}\left(1+\omega_{g}\right)\left(1+\omega_{\tau}\right)},
$$

then $q_{y}>0$ and the objective function is convex. Since the expression on the righthand side of this inequality is necessarily less than one (given that $\Gamma>0$ ), the inequality is satisfied for all values of $s_{G}$ less than a positive upper bound.

\section{A.8 The log-linear aggregate-supply relation and the cost- push disturbance term}

The AS equation (A.61) can be written as

$$
\pi_{t}=\kappa\left[y_{t}+\psi \hat{\tau}_{t}+u_{t}\right]+\beta E_{t} \pi_{t+1}
$$

where $u_{t}$ is composite "cost-push" shock defined as $u_{t} \equiv c_{\xi}^{\prime} \xi_{t}+\hat{Y}_{t}^{*}$. We can write (A.75) as

$$
\pi_{t}=\kappa\left[y_{t}+\psi\left(\hat{\tau}_{t}-\hat{\tau}_{t}^{*}\right)\right]+\beta E_{t} \pi_{t+1}
$$

where we have further defined

$$
u_{t}=u_{\xi}^{\prime} \xi_{t} \equiv \hat{Y}_{t}^{*}+c_{\xi}^{\prime} \xi_{t}
$$

\footnotetext{
${ }^{39}$ The constraint associated with a predetermined value for $Z_{t_{0}}$ can be neglected, in a first-order characterization of optimal policy, because the variable $Z_{t}$ does not appear in the first-order approximation to the aggregate-supply relation.
} 
where

$$
\begin{gathered}
u_{\xi 1} \equiv \frac{\Phi \omega_{\tau}}{q_{y} \Gamma} s_{d}^{-1} \sigma^{-1} \\
u_{\xi 2} \equiv \frac{\Phi \sigma^{-1} s_{d}^{-1} \omega_{\tau}}{q_{y} \Gamma}-\frac{\sigma^{-1} s_{C}^{-1} \Phi\left(1+\omega_{g}+\omega_{\tau}\right)}{q_{y} \Gamma}, \\
u_{\xi 3} \equiv-\Phi \sigma^{-2} \frac{\left(1+\omega_{\tau}\right)\left(1+\omega_{g}\right)}{q_{y} \Gamma\left(\omega+\sigma^{-1}\right)}+\Phi \sigma^{-2} s_{C}^{-1} \frac{1+\omega_{g}+\omega_{\tau}}{q_{y} \Gamma\left(\omega+\sigma^{-1}\right)}, \\
u_{\xi 4} \equiv \omega \sigma u_{\xi 3}, \\
u_{\xi 5}=-\sigma u_{\xi 3}+\frac{(1-\Phi)}{q_{y}},
\end{gathered}
$$

We finally define

$$
\hat{\tau}_{t}^{*} \equiv-\psi^{-1} u_{t}
$$

in a way that we can write (A.61)

$$
\pi_{t}=\kappa\left[\left(\hat{Y}_{t}-\hat{Y}_{t}^{*}\right)+\psi\left(\hat{\tau}_{t}-\hat{\tau}_{t}^{*}\right)\right]+\beta E_{t} \pi_{t+1}
$$

which is equation (2.7) in the text.

\section{A.9 The log-linear intertemporal solvency condition and the "fiscal stress" disturbance term}

The flow budget constraint (A.71) can be solved forward to yield the intertemporal solvency condition

$$
\hat{b}_{t-1}-\pi_{t}-\sigma^{-1} y_{t}=-f_{t}+(1-\beta) E_{t} \sum_{T=t}^{\infty} \beta^{T-t}\left[b_{y} y_{T}+b_{\tau}\left(\hat{\tau}_{T}-\hat{\tau}_{T}^{*}\right)\right]
$$

where $f_{t}$, the fiscal stress disturbance term, is defined as

$$
\begin{aligned}
f_{t} & \equiv \sigma^{-1}\left(g_{t}-\hat{Y}_{t}^{*}\right)-(1-\beta) E_{t} \sum_{T=t}^{\infty} \beta^{T-t}\left[b_{y} \hat{Y}_{T}^{*}+b_{\tau} \hat{\tau}_{T}^{*}+b_{\xi}^{\prime} \xi_{T}\right] \\
& =\sigma^{-1}\left(g_{t}-\hat{Y}_{t}^{*}\right)+(1-\beta) E_{t} \sum_{T=t}^{\infty} \beta^{T-t}\left[\omega_{\tau}^{-1} \Gamma \hat{Y}_{T}^{*}-\left(b_{\xi}^{\prime}-b_{\tau} \psi^{-1} c_{\xi}^{\prime}\right) \xi_{T}\right]
\end{aligned}
$$

This can be rewritten in a more compact way as

$$
f_{t} \equiv h_{\xi}^{\prime} \xi_{t}+(1-\beta) E_{t} \sum_{T=t}^{\infty} \beta^{T-t} f_{\xi}^{\prime} \xi_{T}
$$


where

$$
\begin{aligned}
& h_{\xi 1} \equiv-\frac{\Phi \omega_{\tau}}{q_{y} \Gamma} \frac{\sigma^{-2}}{s_{d}} \\
& f_{\xi 1} \equiv \frac{\Phi}{q_{y}} \frac{\sigma^{-1}}{s_{d}}+\frac{1}{s_{d}}, \\
& h_{\xi 2} \equiv-\frac{\Phi \sigma^{-2} s_{d}^{-1} \omega_{\tau}}{\Gamma q_{y}}+\frac{\sigma^{-2} s_{C}^{-1} \Phi\left(1+\omega_{g}+\omega_{\tau}\right)}{\Gamma q_{y}} \\
& f_{\xi 2} \equiv \frac{\Phi \sigma^{-1} s_{d}^{-1}}{q_{y}}-\frac{\omega_{\tau}^{-1} \sigma^{-2} s_{C}^{-1} \Phi\left(1+\omega_{g}+\omega_{\tau}\right)}{\omega_{\tau} q_{y}}+\frac{1}{s_{d}}, \\
& h_{\xi 3} \equiv \Phi \sigma^{-2} \frac{\left(1+\omega_{\tau}\right)\left(1+\omega_{g}\right)}{q_{y} \Gamma}-\Phi \sigma^{-2} s_{C}^{-1} \frac{1+\omega_{g}+\omega_{\tau}}{q_{y} \Gamma} \\
& +\frac{(1-\Phi) \omega \sigma^{-1}}{q_{y}}+\frac{\omega \sigma^{-1} \Phi(1+\omega)\left(1+\omega_{g}\right)}{\Gamma q_{y}}, \\
& f_{\xi 3} \equiv \frac{\omega_{\tau}^{-1} \Gamma(1-\Phi) \sigma^{-1}}{q_{y}}+\frac{\omega_{\tau}^{-1} \sigma^{-1} \Phi(1+\omega)\left(1+\omega_{g}\right)}{q_{y}}-\sigma^{-1}\left(1+\omega_{\tau}^{-1}\right)\left(1+\omega_{g}\right), \\
& h_{\xi 4} \equiv-\frac{\sigma^{-1}(1-\Phi) \omega}{q_{y}}-\frac{\sigma^{-1} \omega \Phi(1+\omega)\left(1+\omega_{g}\right)}{\Gamma q_{y}}, \\
& f_{\xi 4} \equiv \frac{\omega_{\tau}^{-1} \Gamma(1-\Phi) \omega}{q_{y}}+\frac{\omega_{\tau}^{-1} \omega \Phi(1+\omega)\left(1+\omega_{g}\right)}{q_{y}}-\omega \omega_{\tau}^{-1}\left(1+\omega_{g}\right), \\
& h_{\xi 5} \equiv \sigma^{-1} \Phi \frac{\left(1+\omega_{g}\right)(1+\omega)}{q_{y} \Gamma}, \\
& f_{\xi 5} \equiv-\omega_{\tau}^{-1} \Phi \frac{\left(1+\omega_{g}\right)(1+\omega)}{q_{y}}+\omega_{\tau}^{-1}\left(1+\omega_{g}\right) \text {. }
\end{aligned}
$$

\section{A.10 Definition of the coefficients in sections 3,4 and 5}

The coefficients $m_{\varphi}, n_{\varphi}, n_{b}, m_{b}, \tilde{m}_{b}, \omega_{\varphi}$ are defined as

$$
\begin{aligned}
m_{\varphi} & \equiv-q_{y}^{-1} \psi^{-1}(1-\beta) b_{\tau}+q_{y}^{-1}\left[(1-\beta) b_{y}+\sigma^{-1}\right], \\
n_{\varphi} & \equiv-q_{y}^{-1} \sigma^{-1} \\
n_{b} & \equiv b_{\tau}\left(\psi^{-1}-1\right)\left(m_{\varphi}+n_{\varphi}\right), \\
m_{b} & \equiv-n_{\varphi}\left[(1-\beta) b_{\tau} \psi^{-1}-(1-\beta) b_{y}-\sigma^{-1}\right],
\end{aligned}
$$




$$
\begin{gathered}
\tilde{m}_{b} \equiv \sigma^{-1} n_{\varphi}+\omega_{\varphi}-(1-\beta)\left[b_{\tau} \psi^{-1}-b_{y}\right] n_{\varphi}+(1-\beta) \psi^{-1} \kappa^{-1} b_{\tau} \omega_{\varphi} \\
\omega_{\varphi} \equiv-q_{\pi}^{-1}\left(\kappa^{-1}(1-\beta) b_{\tau} \psi^{-1}+1\right) \\
\phi \equiv \kappa^{-1} q_{\pi}^{-1} q_{y} \\
\gamma_{1} \equiv \kappa^{-1} q_{\pi}^{-1}\left[(1-\beta) b_{y}+\sigma^{-1}\right] \\
\gamma_{2} \equiv \kappa^{-1} q_{\pi}^{-1} \sigma^{-1} .
\end{gathered}
$$

The coefficients $\mu_{1}$ and $\mu_{2}$ of section 5 are defined as

$$
\begin{gathered}
\mu_{1} \equiv \frac{\kappa \psi}{(1-\beta) b_{\tau}+\kappa \psi} \\
\mu_{2} \equiv \frac{\kappa(1-\beta)\left(b_{\tau}-\psi b_{y}\right)}{(1-\beta) b_{\tau}+\kappa \psi} .
\end{gathered}
$$

\section{A.11 Proof of determinacy of equilibrium under the optimal targeting rules}

We now show that there is a determinate equilibrium if policy is conducted so as to ensure that the two target criteria

$$
E_{t} \pi_{t+1}=0
$$

and

$$
\Delta y_{t}+\omega_{\varphi}^{-1}\left(m_{\varphi}+n_{\varphi}\right) \pi_{t}-\omega_{\varphi}^{-1} n_{\varphi} \Delta \pi_{t}=0
$$

are satisfied in each period $t \geq t_{0}$. Note that (A.80) can be written as

$$
\Delta y_{t}=\gamma_{3} \pi_{t}+\gamma_{4} \pi_{t-1}
$$

where

$$
\begin{aligned}
\gamma_{3} & \equiv-\omega_{\varphi}^{-1} m_{\varphi} \\
\gamma_{4} & \equiv-\omega_{\varphi}^{-1} n_{\varphi}
\end{aligned}
$$

Use (A.79), combined with

$$
\tau_{t}-\hat{\tau}_{t}^{*}=\kappa^{-1} \pi_{t}-\psi^{-1} y_{t}-\kappa^{-1} \beta E_{t} \pi_{t+1}
$$


and

$$
E_{t} \Delta y_{t+1}=-\omega_{\varphi}^{-1} n_{\varphi} \pi_{t}
$$

to eliminate $E_{t} \pi_{t+1}, E_{t} y_{t+1}$ and $\tau_{t}-\hat{\tau}_{t}^{*}$ from

$$
\begin{aligned}
\hat{b}_{t-1}-\pi_{t} & -\sigma^{-1} y_{t}+f_{t}=(1-\beta)\left[b_{y} y_{t}+b_{\tau}\left(\hat{\tau}_{t}-\hat{\tau}_{t}^{*}\right)\right] \\
& +\beta E_{t}\left[\hat{b}_{t}-\pi_{t+1}-\sigma^{-1} \hat{y}_{t+1}+f_{t+1}\right] .
\end{aligned}
$$

Then further use (A.80) to eliminate $y_{t}$ from the resulting expression. One obtains an equation of the form

$$
\hat{b}_{t}=\beta^{-1} \hat{b}_{t-1}+m_{41} \pi_{t}+m_{42} \pi_{t-1}+m_{43} y_{t-1}+\varepsilon_{t},
$$

where $\varepsilon_{t}$ is an exogenous disturbance. The system consisting of this equation plus (A.80) and (A.79) can then be written as

$$
E_{t} z_{t+1}=M z_{t}+N \varepsilon_{t}
$$

where

$$
z_{t} \equiv\left[\begin{array}{l}
\pi_{t} \\
\pi_{t-1} \\
y_{t-1} \\
\hat{b}_{t-1}
\end{array}\right], \quad M \equiv\left[\begin{array}{llll}
0 & 0 & 0 & 0 \\
1 & 0 & 0 & 0 \\
m_{31} & m_{32} & 1 & 0 \\
m_{41} & m_{42} & m_{43} & \beta^{-1}
\end{array}\right], \quad N \equiv\left[\begin{array}{l}
0 \\
0 \\
0 \\
n_{41}
\end{array}\right]
$$

Because $M$ is lower triangular, its eigenvalues are the four diagonal elements: $0,0,1$, and $\beta^{-1}$. Hence there is exactly one eigenvalue outside the unit circle, and equilibrium is determinate (but possesses a unit root). Because of the triangular form of the matrix, one can also easily solve explicitly for the elements of the left eigenvector

$$
v^{\prime}=\left[\begin{array}{llll}
v_{1} & v_{2} & v_{3} & 1
\end{array}\right]
$$

associated with the eigenvalue $\beta^{-1}$, where

$$
\begin{gathered}
v_{1}=\left(1+\omega_{g}\right)\left[\psi^{-1}-1\right] \beta \gamma_{4}-\left(1-\beta \sigma^{-1} \gamma_{4}\right)-(1-\beta)\left(1+\omega_{g}\right)(\kappa \psi)^{-1} \\
+\left(1+\omega_{g}\right)\left[\psi^{-1}-1\right] \gamma_{3}, \\
v_{2}=\left(1+\omega_{g}\right)\left[\psi^{-1}-1\right] \gamma_{4}, \\
v_{3}=\left(1+\omega_{g}\right)\left[\psi^{-1}-1\right] .
\end{gathered}
$$


Pre-multiplying the vector equation by $v^{\prime}$, one obtains a scalar equation with a unique non-explosive solution of the form

$$
v^{\prime} z_{t}=-\sum_{j=0}^{\infty} \beta^{j+1} E_{t} \varepsilon_{t+j} .
$$

In the case that $v_{1} \neq 0$, this can be solved for $\pi_{t}$ as a linear function of $\pi_{t-1}, y_{t-1}, \hat{b}_{t-1}$ and the exogenous state vector as it follows

$$
\pi_{t}=-\frac{1}{v_{1}} \hat{b}_{t-1}-\frac{v_{2}}{v_{1}} \pi_{t-1}-\frac{v_{3}}{v_{1}} y_{t-1}+\frac{1}{v_{1}} f_{t} .
$$

The solution for $\pi_{t}$ can then be substituted into the above equations to obtain the equilibrium dynamics of $y_{t}$ and $\hat{b}_{t}$ as well, and hence of $\tau_{t}$ also. 


\section{References}

[1] Aiyagari, S. Rao, Albert Marcet, Thomas Sargent, and Juha Seppala (2002), "Optimal Taxation without State-Contingent Debt," Journal of Political Economy.

[2] Barro, Robert J. (1979), "On the Determination of Public Debt," Journal of Political Economy 87: 940-971.

[3] Benigno, Pierpaolo, and Michael Woodford (2003), "Inflation Stabilization and Welfare: The Case of Large Distortions," in preparation.

[4] Bohn, Henning (1990), "Tax Smoothing with Financial Instruments," American Economic Review 80: 1217-1230.

[5] Chari, V.V., Lawrence Christiano J., and Patrick Kehoe (1991), "Optimal Fiscal and Monetary Policy: Some Recent Results," Journal of Money, Credit, and Banking 23: 519-539.

[6] Chari, V.V., Lawrence Christiano J., and Patrick Kehoe, (1994) "Optimal Fiscal Policy in a Business Cycle Model, " Journal of Political Economy 102: 617-652.

[7] Chari, V.V., and Patrick J. Kehoe (1999), "Optimal Fiscal and Monetary Policy," in J.B. Taylor and M. Woodford, eds., Handbook of Macroeconomics, vol. 1C, North-Holland.

[8] Clarida, Richard, Jordi Gali, and Mark Gertler (1999), "The Science of Monetary Policy: a New Keynesian Perspective," Journal of Economic Literature, 37 (4), pp. 1661-1707

[9] Correia, Isabel, Juan Pablo Nicolini and Pedro Teles (2001), "Optimal Fiscal and Monetary Policy: Equivalence Results," unpublished manuscript, Bank of Portugal.

[10] Friedman, Milton (1969), "The Optimum Quantity of Money," in The Optimum Quantity of Money and Other Essays, Chicago: Aldine.

[11] Giannoni, Marc, and Michael Woodford (2002), "Optimal Interest-Rate Rules: I. General Theory," NBER working paper no. 9419, December. 
[12] Giannoni, Marc, and Michael Woodford (2003), "Optimal Inflation Targeting Rules," in B.S. Bernanke and M. Woodford, eds., Inflation Targeting, Chicago: University of Chicago Press, forthcoming.

[13] Hall, George, and Stefan Krieger (2000), "The Tax Smoothing Implications of the Federal Debt Paydown," Brookings Papers on Economic Activity 2: 253-301.

[14] Khan, Aubhik, Robert G. King, and Alexander L. Wolman (2002), "Optimal Monetary Policy," NBER working paper no. 9402, December.

[15] Lucas, Robert E., Jr., and Nancy L. Stokey (1983), "Optimal Fiscal and Monetary Policy in an Economy without Capital," Journal of Monetary Economics 12: $55-93$.

[16] Rotemberg, Julio J., and Michael Woodford (1997), "An Optimization-Based Econometric Framework for the Evaluation of Monetary Policy", in B.S. Bernanke and Rotemberg (eds.), NBER Macroeconomic Annual 1997, Cambridge, MA: MIT Press. 297-346.

[17] Sbordone, Argia M. (2002), "Prices and Unit Labor Costs: A New Test of Price Stickiness," Journal of Monetary Economics 49: 265-292.

[18] Schmitt-Grohé, Stephanie, and Martin Uribe (2001), "Optimal Fiscal and Monetary Policy under Sticky prices," Rutgers University working paper no. 2001-06, June.

[19] Siu, Henry E. (2001), "Optimal Fiscal and Monetary Policy with Sticky Prices," unpublished manuscript, Northwestern University, November.

[20] Svensson, Lars E.O. (1999), "Inflation Targeting as a Monetary Policy Rule," Journal of Monetary Economics 43: 607-654.

[21] Svensson, Lars E.O. (2003), "What Is Wrong with Taylor Rules? Using Judgment in Monetary Policy through Targeting Rules," Journal of Economic Literature, forthcoming.

[22] Svensson, Lars E.O., and Michael Woodford (2003), "Implementing Optimal Policy through Inflation-Forecast Targeting," in B.S. Bernanke and M. Woodford, eds., Inflation Targeting, Chicago: Univ. of Chicago Press, forthcoming. 
[23] Woodford, Michael (1999), "Commentary: How Should Monetary Policy Be Conducted in an Era of Price Stability?" in Federal Reserve Bank of Kansas City, New Challenges for Monetary Policy.

[24] Woodford, Michael (2001), "Fiscal Requirements for Price Stability," Journal of Money, Credit and Banking 33: 669-728.

[25] Woodford, Michael (2003), Interest and Prices: Foundations of a Theory of Monetary Policy, forthcoming, Princeton University Press. 